\title{
THE USE OF THE POTENTIAL DROP TECHNIQUE FOR CREEP DAMAGE MONITORING AND END OF LIFE WARNING FOR HIGH TEMPERATURE COMPONENTS
}

\author{
A. Wojcik (corresponding author) \\ Department of Mechanical Engineering, University College London, UK \\ a.wojcik@ucl.ac.uk \\ M. Waitt
}

A. S Santos

Both of Matelect Ltd, Harefield, UK 


\title{
THE USE OF THE POTENTIAL DROP TECHNIQUE FOR CREEP DAMAGE MONITORING AND END OF LIFE WARNING FOR HIGH TEMPERATURE COMPONENTS
}

\author{
The electrical potential drop (EPD) technique is well-established for the \\ measurement of crack initiation/growth in metals. Two variants exist (using AC \\ or DC excitation current). EPD is a powerful way to gauge crack dimensions \\ (principally depth) in a variety of contexts, including laboratory based \\ fracture/fatigue testing, and in-field NDE, however whilst it has been used for on \\ and off-line assessment of creep damage, the use within a non-lab based (i.e. \\ industrial) context is fraught with difficulties including methodology and \\ connection issues and, significantly, data interpretation - especially with regard \\ to detecting subtle changes in EPD over a general background "noise" whose \\ magnitude often exceeds the changes due to creep damage (until a defect is so \\ large that failure is imminent). We describe, here, a method where high \\ sensitivity detection of creep damage can be achieved by the simple expedient of \\ looking for a characteristic "signature" in the data sets. This methodology has \\ been strengthened by the combination of AC based EPD with its DC equivalent, \\ such that both techniques generate a synergistic approach to the detection of \\ creep damage. The methodology has been successfully applied to a semi- \\ industrial on-line context to provide prior warning of failure of several weeks.
}

Keywords: electrical potential drop, EPD, ACPD, DCPD, creep, on-line monitoring

\section{Introduction}

The electrical potential drop (EPD) technique is well-established for the measurement of crack initiation/growth in metals. The DC variant (DCPD), developed over 50 years ago, was joined by the AC equivalent (ACPD) in the 1980's [1].

EPD (also known simply as Potential Drop, PD) remains a powerful way to gauge crack dimensions (principally depth, but also surface breaking width and even inclination) in a variety of contexts, including laboratory based fracture/fatigue testing 
[2], in-field NDE, and on-line continuous monitoring. Modern EPD equipment offers high resolution (often to microns of crack growth), sophisticated noise reduction, and multichannel capability, within a compact, expandable, fully digital system.

The use of EPD for creep based studies is not new, and relies upon the fact that many of the factors associated with creep in metals, such as the development of plastic strain, and the formation of cavities and their eventual coalescence to generate demonstrable crack-like defects, affect the EPD signals in much the same way that a developing crack would. However EPD usually comes into its own when most of the creep lifetime has been expended, and can show poor sensitivity to the earlier stages of creep damage development. Additionally, the transfer of laboratory based (i.e. well controlled) EPD set-ups to the more practical environments inherent in on-line plant life monitoring is fraught with difficulties, not least of which is interpreting the signals. Changes in the EPD can be generated by factors in the test environment that have little do with actual creep damage, such as those due to variations in test conditions (typically temperature and/or applied load). These changes can be orders of magnitude greater than those that are expected via creep damage alone.

This paper details some of the EPD work carried out as part of a series of long term creep tests performed on P91 and P92 pressure vessels. This is turn was part of a wider study aimed at better lifetime prediction for components manufactured from these materials and utilized in power generation applications. 
This paper reports on many of the practical issues and the lessons learned in undertaking this work, and in particular discusses the feasibility of applying the EPD technique into a real-life industrial on-line monitoring context.

\section{Background}

In its most basic form, EPD relies upon a measurement of a specimen's electrical impedance. In the case of DCPD, this is specifically the electrical resistance, whereas in ACPD, capacitive and inductive components complement the electrical resistance to generate a more complex parameter [3].

Conventionally, impedance is measured using a four point arrangement of inline electrical contacts, with the outer two connections delivering the excitation current, and the inner two allowing measurement of the potential drop required to drive the excitation current through the specimen (this being a function of the impedance). The method relies upon the influence of a defect on the specimen's impedance. Normally crack-like defects act to raise the local impedance, and therefore can be detected by a rise in the local EPD. Measurements are normally simplified by ensuring that the excitation current is known and remains constant at least throughout the duration of the measurement.

A further subtlety of ACPD over DCPD is the existence of the so-called skin effect, where the excitation current is found to travel close to the surface of the specimen, rather than uniformly throughout its cross-section (this being largely the case for DCPD). A practical consequence of this phenomenon is that the calibration 
methodology (EPD vs crack depth) is different for ACPD compared to DCPD.

Additionally the skin effect (specifically the depth that most of the current penetrates to - the skin depth) is a function of the frequency of the AC excitation, and this provides ACPD with an extra degree of freedom which can both add information or complicate interpretation (depending on one's viewpoint). The higher the frequency, the smaller the skin depth, and the more sensitive the technique is to surface breaking defects.

Many of the practical challenges associated with EPD relate to the engineering difficulties in maintaining a constant current, and also the way in which electrical connections are made to specimens [3]. However, EPD excels at providing a continuous electrical response proportional to crack dimensions and as such it is often the only crack monitoring technique that can be used in extreme testing contexts such as at high temperatures (e.g. thermomechanical testing of superalloys) [4], under corrosive atmospheres (e.g. $\mathrm{H}_{2} \mathrm{~S}$ induced cracking in the oil and gas industry, or stress corrosion cracking of stainless steels under high pressure high temperature aqueous conditions) [5], or even in high radiation environments (such as in-pile testing of materials in the nuclear industry). In such contexts EPD is normally used in a continuous (on-line) sense to monitor for crack initiation and crack growth.

For NDE applications, EPD equipment can be battery powered and then used for spot-checking of cracks in structures (particularly ACPD, given it's better portability), with the 4 point connections being made by some kind of position-able or hand-held "probe" head which usually houses sprung loaded pins able to penetrate surface oxides or contamination. However, resolutions reached (in terms of crack depth) are nothing like that achievable in an on-line context. This is as a result of several reasons. Very 
few commercial hand-held crack depth "meters" have ever employed DCPD as the measurement method, simply because most metallic specimens are very effective short circuits, so the excitation currents have to be in the 10's or even 100's of amps before a decent measureable DCPD can be obtained - and passing such high currents through sprung pins is not a very long term proposition. Therefore any DC based system is likely to be of poor sensitivity as a consequence of the low maximum current capability.

Conversely, handheld ACPD probes are very susceptible to registering changes in signal magnitude due to differences in approach angle and contact pressure. These effects are linked to the existence of an error signal, that superimposes upon the specimen-derived signal, and is generated as a consequence of induction phenomenon (much like lift-off in Eddy current based NDE). The resultant variability inevitably affects resolution.

Such effects generally limit handheld EPD to a resolution of no better than $0.5 \mathrm{~mm}$ in crack depth measurements - a far cry from the 10 micron resolution that is normally achievable for typical cracks in a continuously monitored situation [4]. Not only does this limit the utility of EPD based NDE of cracks, it does preclude probe based measurement of a range of other effects that might be of interest (and which have been shown to influence EPD readings) including microstructural differences (such as weld HAZs, or case hardening), internal defects (e.g. porosity in castings), residual stress measurements (ACPD is sensitive to the level of elastic and plastic strain in ferrous materials), and of course creep damage, such as the development of cavitation. 
EPD seems to have been rarely used for on-line use, and this may have something to do with the practicalities of making robust connections in the field as well as interpreting the data generated. In this study, many of the practical issues associated with specimen connection and apparatus deployment have been overcome - certainly to the extent which no longer requires a laboratory based or academic approach to be undertaken. Data interpretation remains a challenge, although as will be described here, initial results of the work on this look promising. Part of the advance has been to combine $\mathrm{AC}$ and $\mathrm{DC}$ variants of the EPD technique into one monitoring system to effectively create a "ACDC"-EPD set-up. Not only has this enabled the benefits and strengths of both variants to be captured in one on-line test, it has also revealed a synergistic effect which appears to greatly enhance an operator's ability to determine (in this case) how close to end-of-life a monitored component is.

\section{Practical details}

The ACDC set-up employed in this study was created by interfacing two commercial EPD instruments together, one providing ACPD capability and the other, DCPD, (Matelect Ltd, London). This was achieved by using a series of signal and current multiplexing (switching) units which also facilitated connection to multiple points on the test vessel. Several cylindrical pressure vessels were monitored, in a three year study. Figure 1 shows one of these - manufactured from P91 steel and containing two circumferential welds. The study aimed to investigate creep damage development, and to characterize this using a variety of non-destructive methods. The vessel was mounted vertically (Figure 1) and subjected to both elevated temperature (ca 700 DegC), internal pressure, and external axial load. The vessel was loaded to levels which, at the test temperature, were deemed to result in failure via creep in around 10k 
hours. Failure was expected to initiate in the HAZ of the welds by Type IV cracking and it was these zones that were monitored by the EPD system. Scheduled outages were also planned (as part of the wider study) so that an array off-line NDE characterisation methods could be employed. This included ACPD (in a hand-held mode), but this paper only discusses on-line results, as this methodology will ultimately prove more useful to an industrial user, than an outage based assessment of lifetime.

A significant feature of the ACDC system was the ability to employ only one set of electrical connections for both EPD variants. This both simplified connections and reduced the number of wires and connection points to the test vessels, the only disadvantage being that the ACPD current carrying wires were, in effect, much thicker than they would normally be (given they also had to be capable of carrying the higher direct currents for DCPD).

After a considerable period of trial and error, a connection methodology which involved the use of stainless steel studs (ca 20mm long x $2 \mathrm{~mm}$ diameter) silver-soldered to pure silver wire, the whole being sheathed in silica braiding, was eventually employed. The studs were welded to the vessels using a conventional spot welder, operated as a stud welder.

Studs were pre-tinned to assist subsequent soldering, and in some cases, they were also pre-soldered to short $(100 \mathrm{~mm})$ lengths of silver wire, which proved easier to join in the field to the main silver leads, especially where access was limited. The silica braiding was applied to pre-measured lengths of silver wire before soldering. Wire of $1.5 \mathrm{~mm}$ diameter was used for the current supply lines, and 0.5 for the signal lines. 
Typical lead lengths were 3 metres from connection point on the test vessel to a nearby junction block (external to the furnace), whereupon connection to the multiplexers (and thence to the AC and DC instruments) was via shielded twisted pair copper cable. Wherever possible (and to help eliminate interference or "pick-up" in ACPD situations) all wire pairs (signal as well as current) were twisted together, although this was minimised to avoid damaging the silica braid. A weld contains two adjacent HAZ locations, and therefore each HAZ was monitored separately. A total of 6 studs were positioned in-line across a weld therefore, with the outer two delivering the requisite EPD excitation current, with the two inner pairs, straddling each HAZ, acting as the EPD measurement points. Several "zones" along any one weld were covered, and the complete ACDC system contained 18 separate EPD measurement points per vessel.

Part of this set-up and the P91 test vessel can be seen in Figure 1.

Silver proved an inspired choice, being extremely easy to handle in the field, thread through the braid, and solder in place. It resisted oxidation admirably, and even after 10,000 hours of exposure, was always bright and free from oxidation, unlike the stainless studs which became heavily oxidized. Connection failures were common at the start of the work, but once the pre-tinning was introduced, and the stud welding parameters optimized, these disappeared. Use of standard fluoride based fluxes for insitu soldering did give rise to degradation of the silica braid if residues were not scrupulously washed off, but with care, and the use of pre-tinned studs (which largely precluded in-situ fluxing), this problem was eliminated. Results using nickel wire were nowhere near as reliable and the higher electrical resistance of nickel required thicker wires to be employed (for the current supply leads) which hampered installation. 
The ACDC instrumentation was placed external to the furnace and blast zone, and the whole EPD system was placed under the control of bespoke software which could be accessed across the Net, and permitted the easy transfer of data for regular interpretation.

For the ACPD aspect, skin depth calculations for P91 steel at the frequencies available suggested that even at the lowest operating frequency of $300 \mathrm{~Hz}$, the skin was substantially thinner than the specimen wall thickness (ca $5 \mathrm{~mm}$ compared to $25 \mathrm{~mm}$ ). This meant that the ACPD readings were only expected to reveal defects and/or microstructural variations that were close to the outer surface of the pipe specimen. Creep cavitation could extend to the surface regions but past experience suggested that crack development would initially be internal before travelling to either the outside surface or the inside (back-face). The lowest excitation frequency $(300 \mathrm{~Hz})$ was therefore expected to give the best chance of showing any internal or back-face defect.

The ACPD system utilized was capable of providing both real and imaginary components of the ACPD reading. AC signals contain both phase and amplitude information, hence can be resolved into vectors representing the real (i.e. resistive) and the imaginary (capacitive and inductive) components of the signal. The resistive component is normally related to crack depth through a linear or near-linear relationship, whereas the imaginary component tends to be ignored in conventional ACPD. The imaginary component can however be used to help optimise the measurement set-up. If the values of the imaginary components are large, then this 
casts doubt upon the validity of the resistive components as saturation of the amplifiers in the ACPD system may well be occurring.

The AC excitation current was set to 2 amps for all measurements. In contrast, direct currents of ca 50A are sometimes required for comparable signal magnitudes, but this is often not possible using commercial equipment if the lead lengths are long (and hence the overall resistance of the current path). High currents can also cause specimen heating and this can (and did) lead to drift in DC signals if currents higher than 15A were employed. A similar set-up was employed for the P92 vessel that was an additional part of the study, the only differences being that this vessel was mounted horizontally, and no external axial load applied. Also, temporally, the P92 tests came after the P91 tests.

\section{Results and implications}

Testing of this kind creates vast amounts of data, and detailed analysis is not attempted here, however broad lessons for creep crack development and subsequent monitoring can be drawn, and will prove valuable to future studies. Figure 2 shows a plot of data extracted from a representative data file and illustrates a typical DCPD response over a total period of just over 2.5 months from an adjacent pair of HAZ locations (designated "x" and "y") on the P92 vessel.

It can be readily seen that there is much variation in signal magnitudes over time. Note that the complimentary ACPD response would be equally as "busy". 
The fluctuations seen amount to several 10's of \% of full scale, and this is far in excess of any fluctuation expected due to crack initiation. Once a crack had initiated and grown to be a substantial fraction of the specimen wall thickness, such a change in signal level might be expected, and indeed exceeded, but clearly the observed fluctuations in Figure 2 recover in magnitude and level, and are definitely, therefore, not due to specimen cracking. Furthermore such changes were often observed early on in the projected lifetime of the vessel, so could not be reasonably ascribed to cracking.

Most of these fluctuations were traced to changes in specimen temperature. The two large dips (note dips, not rises as would be expected if a crack had developed) were actually associated with a total failure of the furnace temperature control and subsequent shutdown of heating.

In laboratory based EPD, during elevated temperature testing, it is normal to employ a reference channel to normalise for such temperature fluctuations. A ratio of active/reference EPD is then recorded and this should be immune from changes in temperature. Normalisation does indeed generate a far quieter response, however as Figure 3 shows, a simple arithmetic normalisation (in this case the signal from one HAZ (x) divided by the signal from the adjacent HAZ (y)) can still be fooled.

The presence of the transient in the normalised signal can only be explained if the effect of temperature on the signals is non-linear and/or the shutdown affected the signals via other, additional, (and non-linear) factors, such as a change in stress. 
The above issue highlights the difficulty and challenges that technicians will face when using EPD for on-line monitoring. Other signals (such as temperature) clearly need to also be monitored and interpreted alongside the EPD data, if any certainty is to arise in practice.

Irrespective of the above, it will still be necessary to "extract" a signal from such a response that in some way represents the information on creep behaviour. Once a transient can be explained in terms of an external factor, it is not unreasonable to simply delete the transient data, and re-join the ends of the cleaned response together. Other filtering methods can be employed to remove solitary transients which are clearly "rogue" points and act to mask the longer term trends. Figure 4 illustrates what can be done using this approach, to the data from the P92 vessel.

The data is now far noisier than before, as autoscaling has been employed to essentially raise signal gain (possible now that the transients have been removed). The scale now reveals that noise in the EPD is at a level of 10's of nanovolts - and hence is more likely to be a reflection of overall instrumental noise. Emerging from this noise can be seen a clear trend however - a gentle but steady rise in the EPD which is much more likely to be as a result of the development of creep damage than to "external" influences. Given that this data set is obtained from a zone on the P92 vessel directly over where final rupture occurred, more will be said about this rise later.

In the present study, once such data processing was performed on the $\mathrm{AC}$ and DC signals, further trends were identified, which helped to indicate that creep damage had reached the late stages in a specimen's lifetime, where cracking was expected. 
Figure 5 reveals what was observed to happen to the EPD once a defect had initiated in one of the circumferential welds, and was actively growing, in the P91 vessel. The presence of the crack was confirmed after catastrophic failure of the vessel had occurred. In this case, a rise in EPD was not being employed as an early predictor of impending failure, but an examination of the trends seen revealed a pattern of responses that was not entirely as expected.

It should be noted that, as before, two signals were being monitored at this zone, namely one from either side of the weld, so that both HAZ were covered (also AC and DC). The DC trends appear to work in opposition to each other with the one HAZ zone showing a clear exponential rise associated with a rapidly propagating defect, but the complimentary HAZ trace shows a gentle decline. The explanation for this is easily understood if it is noted that both monitored zones are being fed the same excitation current and lie in line with each other (in terms of current flow) hence the growing defect will a) raise the DCPD (in the expected sense) and b) divert the current flow away from the adjacent zone such that it appears to show a reduction in measured DCPD.

It was clear that the change in the DCPD was very dramatic and highly definitive, when in the latter stages of failure (the last two days), but that nevertheless, a far subtler rise was seen at least two weeks ahead of failure. Given the comments made earlier, such a rise would have been insufficient to provide an operator with any great certainty of impending failure - at least until a few days before it occurred. 
However, when considered together with the drop seen in the adjacent HAZ's response, a greater degree of certainty could be assumed whenever a similar "pattern" is observed.

Further to this, Figure 6 illustrates the corresponding ACPD traces, for the same locations on the P91 vessel, and here it can be seen that as the DC traces rise, the AC responses drop. Unlike for DCPD, this occurs on both HAZ positions and the drop continues until such time as the DC trace has begun its exponential rise, at which point the $\mathrm{AC}$ response also turns and appears to follow suit by rising almost vertically (signifying rapid crack propagation).

The explanation for the $\mathrm{AC}$ behaviour is not obvious and relies upon the knowledge that in ferritic materials, ACPD signals are sensitive to stress (strain in reality) [1]. This is a result of the change in magnetic permeability that occurs when the grains containing the magnetic domains are strained. As a result, in a ferritic material under a uniaxial stress, the ACPD measured axially has been observed to drop as the stress rises.

In the monitored pressure vessel, the developing defect will be expected to raise the local stress to a point where the ACPD may indeed be affected by the stress concentration.

Given that the "true" ACPD is normally only sensitive to surface breaking defects (as it relies upon the skin effect to generate a rise in path length as a defect 
grows) it is likely that the $\mathrm{AC}$ response will first drop before finally rising (presumably once the defect has become surface breaking).

When taken together, the drop in $\mathrm{AC}$ with the rise in $\mathrm{DC}$, and the drop in $\mathrm{DC}$ on one HAZ, with a rise in the other, constitute a characteristic "signature" which could greatly lengthen the warning period ahead of an impending failure.

After this initial success, the failed weld in the P91 vessel was removed and the shortened vessel was placed back under test, with the aim of enabling the second weld to fail. The ACDC EPD monitoring system was re-instigated with fresh connections around this remaining weld. Similar trends were noted in some of the monitored locations in this second test and, as these trends were developing in time, a decision was taken to eventually stop the P91 creep test ahead of a scheduled outage. No surface breaking defect was observed (and none was found subsequently in the internal surfaces of the vessel), but ultrasound inspection (UT) was implemented and confirmed the existence of sub-surface cracking. Subsequent sectioning and metallographic examination has supported the UT and EPD results, and has revealed that as well as an internal crack, substantial cavitation damage had occurred in the vicinity of the developing defect. However the possibility remains that the cavitation is an effect of, rather than the cause of, cracking.

Conservative estimates of the length of warning that the EPD gave the test operators of a leakage in the P91 vessel, via a surface breaking defect, are in the region of two to three weeks. This may well be enough for many plant operators. 
Unfortunately, the difficulty with definitively ascribing changes in the observed EPD to creep damage per se, (i.e. other than to cracking, as initiated, through creep), is that none of the tests conducted managed to catch a test vessel before evidence of cracking had developed (albeit internal cracking). Thus, maintaining that EPD can detect creep damage (as distinct from cracking), such as a rise in local strain and/or the development of cavitation, is easy to challenge. In truth, whilst the ability to see such damage is clearly of value to metallurgists and researchers alike, its value to plant operators is probably less than a reliable warning of impending vessel failure. Nevertheless, in defence of the belief that EPD can indeed be sensitive to pre-crack damage, Figure 4 should be recalled.

The subtle (relatively), but steady, rise in DCPD in Figure 4 was actually present for some 2.5 months (ca. 1600 hours) before the test was terminated and the P92 test vessel examined using UT. No crack-like defects were detected, the EPD connections remade, and the vessel was put back under test. The gentle rise in DCPD was observed to continue (at a similar, almost linear, gradient) until final cracking and failure occurred some 2 months later, whereupon a rapid rise in DCPD was observed (similar to that seen in the earlier P91 tests). The percentage change in DCPD amounted to less than a $1.5 \%$ rise over the initial 4.5 months, whereas the change in the last few hours of life amounted to over $100 \%$. It is highly unlikely that a crack-like defect existed 4.5 months prior to failure, so the subtle change in DCPD may well have been due to "other" forms of creep induced damage, for example cavitation.

The longevity, expense, and complexity of the vessels tests limited the ability to reproduce these observations, but of the three tests conducted to failure (2x P91 and 1x 
P92), all three have shown modest initial rises in DCPD readings close to the ultimate failure location. Obviously, well defined and constructed laboratory tests would provide a better understanding of the influence of creep on the EPD response in P91/P92, in contrast to the real-word tests conducted here, but this should not detract from the positive nature of the results.

\section{Conclusion}

We present here encouraging results for a modern manifestation of a traditional technique. The EPD work carried out as described has led to a substantial improvement in the practical knowledge of how an EPD based monitoring system should be implemented on an "industrial" creep test specimen, in particular, for helping to detect the later stages of life and the onset of final fracture and cracking. It is envisaged that transferring this experience and methodology to in-situ testing of power station components in service would not pose too great a challenge.

What has always been presented as a challenge has been the interpretation of the results, however. This has traditionally been the domain of the scientific researcher rather than staff working in an engineering and maintenance context. This is due to the complexity of the signal responses and the likelihood that changes in signal can be ascribed to a range of phenomena, only some of which have anything to do with creep damage.

This situation has not been totally eliminated by the current work, but the emergence of the notion that a combination of ACPD and DCPD can help in interpreting creep behaviour, is a definite step forward. This notion was based on the 
idea that a developing defect, such as a crack, will increase specimen resistance (hence raising the $\mathrm{PD}$ ) in the case of $\mathrm{DCPD}$, but could also raise section stresses (due to the development of stress concentrations) hence lowering ACPD (up to a point, and then the PD would rise, as the contribution from the defect began to dominate).

Of note in the case of DCPD, was a further synergistic effect gained by the use of pairs of signal pick up points (one for each HAZ of a monitored weld), which revealed that as the one straddling the developing defect increased, the one on the complimentary HAZ reduced. This can be explained by the developing defect's additional ability to redirect the flow of excitation current away from the second HAZ measurement point. The consequence of a lower current density was a lower signal magnitude - so as one signal rose, the other fell.

In addition to helping interpret the on-line results, the idea of a "signature" for defect detection, if correct, could allow earlier detection - much earlier that would be the case if a single EPD response was being monitored.

It should be noted that the signature described here is particularly confined to a double HAZ situation in a weld in a ferritic material, however it is feasible to suggest that other signatures can be identified and checked against, in other testing contexts.

Of further note were EPD observations that were made well before ultimate failure of the test vessels. These showed that once larger transients were stripped out, locations where failure subsequently developed did show subtle, but definite, changes in EPD months prior to rupture. In particular, DCPD responses were seen to rise in a linear 
fashion for up to 4.5 months before final fracture. The challenge now is to catch another vessel "in the act" well before any demonstrable cracking has developed.

\section{Acknowledgements.}

The authors would like to thank Dr A Shibli and Dr D Robertson of ETD Consulting of Leatherhead UK for their help and support in undertaking the work described here and MPA Stuttgart for access to their facilities and universally helpful staff (especially Dr A Klenk and Dr A Hobt).

\section{References}

1. Venkatsubramanian, T. V., Unvala, B. A, 1985, An AC potential drop system for monitoring crack length, Journal of Physics E: Scientific Instruments, 17(9) pp765-771

2. Gibson, G. P., 1987, The use of alternating current potential drop for measuring Jcrack resistance curves, Engineering Fracture Mechanics 26(2) pp213-222

3. Annex A6, 2015, Guidelines for electrical Potential difference determination of crack size, ASTM E647-15e1 Standard Test Method for Measurement of Fatigue Crack Growth Rates, ASTM International

4. Marchand, N. J., Dorner, W., \& Ilschner, B., 1990, A Novel Procedure to Study Crack Initiation and Growth in Thermal Fatigue Testing, ASTM STP 1060 pp237-259. 
5. Fanjiang, M., Lu, Z., Shoji, T., Wang, J., Han, E., Ke, W., 2011, Stress corrosion cracking of uni-directionally cold worked $316 \mathrm{NG}$ stainless steel in simulated PWR primary water with various dissolved hydrogen concentrations, Corrosion Science 53 pp2558-2565 


\section{Figures}

Figure 1. In-situ set-up on the P91 test vessel (EPD leads are visible as white wires exiting to lower RHS).

Figure 2. Typical DCPD plot for period of approximately 3 months showing massive fluctuations associated with changes in thermal conditions. Data is for two adjacent but in-line HAZ monitoring zones, designated " $x$ " and " $y$ " on the P92 vessel.

Figure 3. Normalised DCPD plot for data presented in Figure 2 (thus ratio HAZx/HAZy) which should have eliminated any changes due to temperature fluctuations, but clearly has not.

Figure 4. Processed DCPD plot for early stage data from the P92 vessel (HAZy only) at the location where final failure occurred. Transients have been removed and the vertical scale expanded. Long term trends can now be seen at or close to the intrinsic noise level of the technique, and are several orders of magnitude smaller than transients seen previously.

Figure 5. DCPD plot for zone containing a developing creep crack on the P91 vessel of Figure 1. Rising curve was for crack site HAZ, whereas falling curve for adjacent noncracked HAZ. (x-axis represents 1 month approx.).

Figure 6. Corresponding ACPD plots for zone in Figure. 5 containing a creep crack. Both traces steadily drop (ignoring transients) until crack rapidly propagates to final rupture. 
Fig1

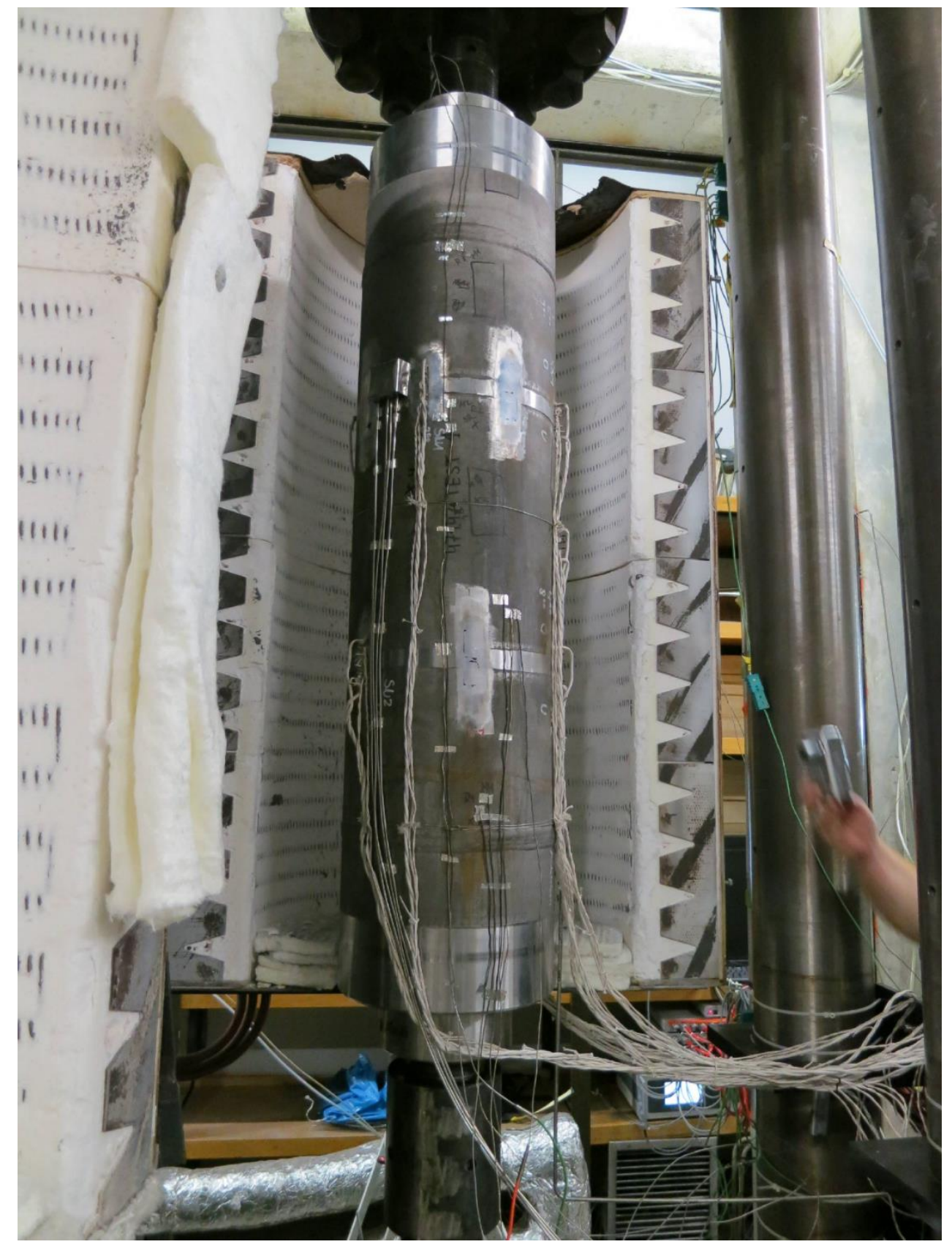


Fig 2

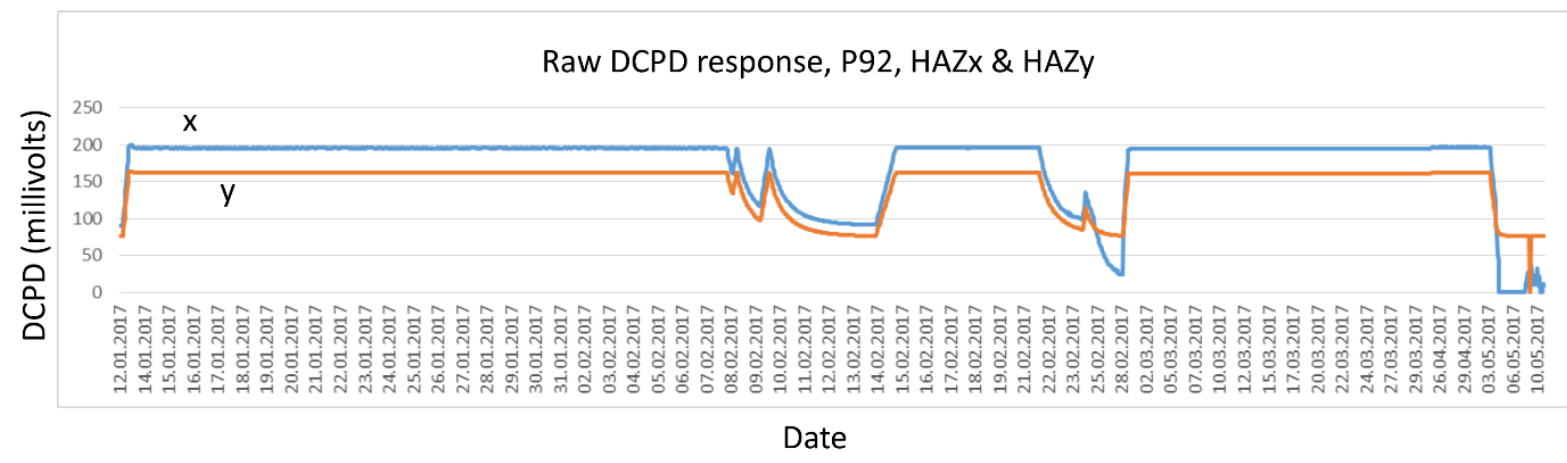

Fig 3.

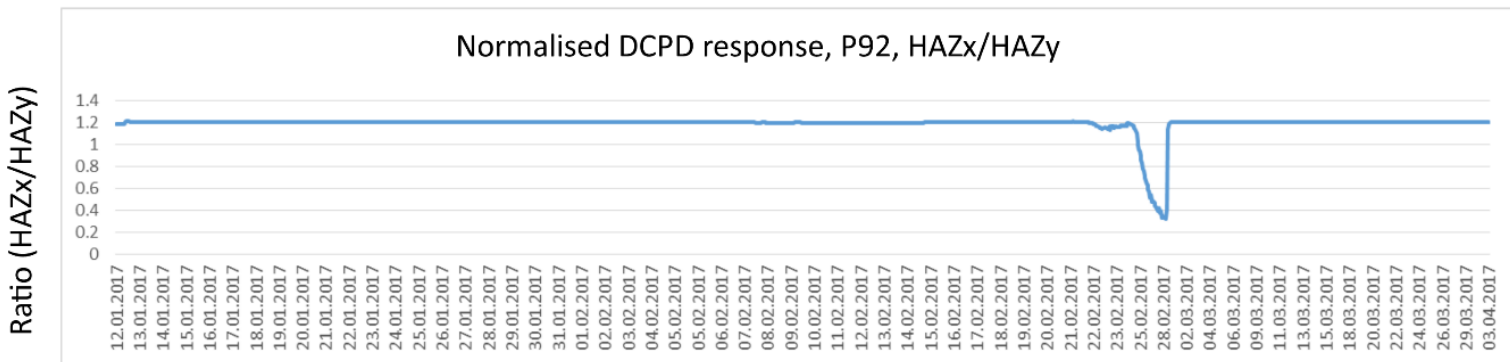

Date

Fig 4.

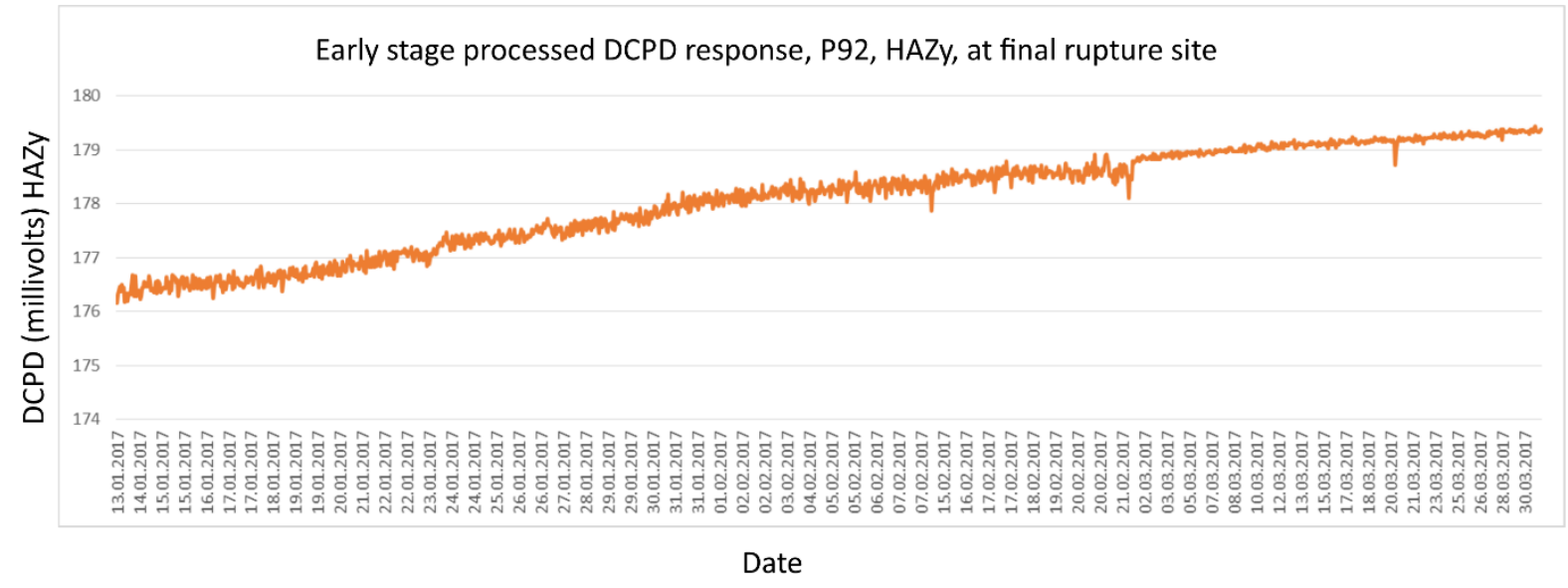


Fig 5.

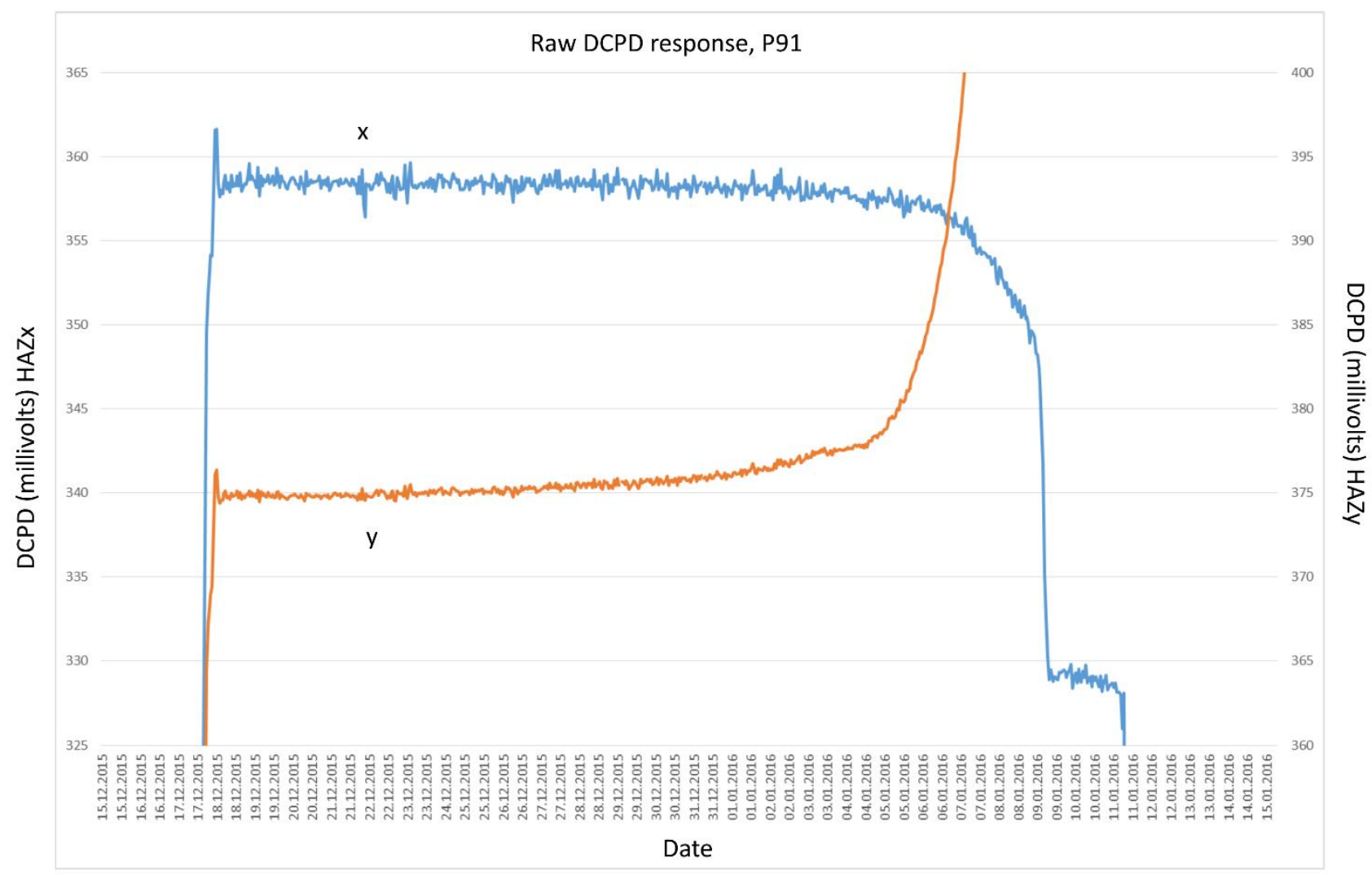


Fig 6.

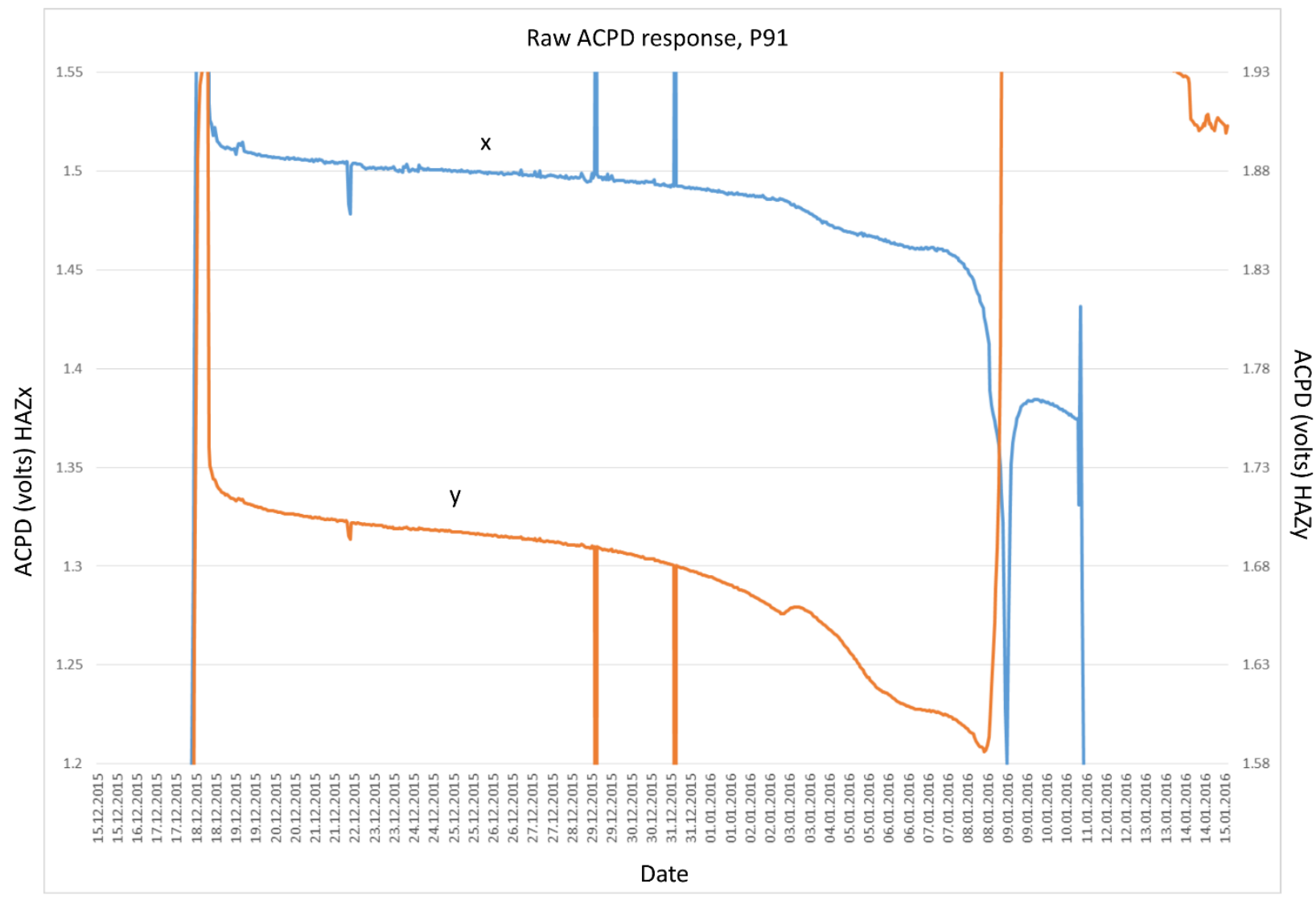




\title{
THE USE OF THE POTENTIAL DROP TECHNIQUE FOR CREEP DAMAGE MONITORING AND END OF LIFE WARNING FOR HIGH TEMPERATURE COMPONENTS
}

\author{
A. Wojcik (corresponding author) \\ Department of Mechanical Engineering, University College London, UK \\ a.wojcik@ucl.ac.uk \\ M. Waitt
}

A. S Santos

Both of Matelect Ltd, Harefield, UK 


\title{
THE USE OF THE POTENTIAL DROP TECHNIQUE FOR CREEP DAMAGE MONITORING AND END OF LIFE WARNING FOR HIGH TEMPERATURE COMPONENTS
}

\author{
The electrical potential drop (EPD) technique is well-established for the \\ measurement of crack initiation/growth in metals. Two variants exist (using AC \\ or DC excitation current). EPD is a powerful way to gauge crack dimensions \\ (principally depth) in a variety of contexts, including laboratory based \\ fracture/fatigue testing, and in-field NDE, however whilst it has been used for on \\ and off-line assessment of creep damage, the use within a non-lab based (i.e. \\ industrial) context is fraught with difficulties including methodology and \\ connection issues and, significantly, data interpretation - especially with regard \\ to detecting subtle changes in EPD over a general background "noise" whose \\ magnitude often exceeds the changes due to creep damage (until a defect is so \\ large that failure is imminent). We describe, here, a method where high \\ sensitivity detection of creep damage can be achieved by the simple expedient of \\ looking for a characteristic "signature" in the data sets. This methodology has \\ been strengthened by the combination of AC based EPD with its DC equivalent, \\ such that both techniques generate a synergistic approach to the detection of \\ creep damage. The methodology has been successfully applied to a semi- \\ industrial on-line context to provide prior warning of failure of several weeks.
}

Keywords: electrical potential drop, EPD, ACPD, DCPD, creep, on-line monitoring

\section{Introduction}

The electrical potential drop (EPD) technique is well-established for the measurement of crack initiation/growth in metals. The DC variant (DCPD), developed over 50 years ago, was joined by the AC equivalent (ACPD) in the 1980's [1].

EPD (also known simply as Potential Drop, PD) remains a powerful way to gauge crack dimensions (principally depth, but also surface breaking width and even inclination) in a variety of contexts, including laboratory based fracture/fatigue testing 
[2], in-field NDE, and on-line continuous monitoring. Modern EPD equipment offers high resolution (often to microns of crack growth), sophisticated noise reduction, and multichannel capability, within a compact, expandable, fully digital system.

The use of EPD for creep based studies is not new, and relies upon the fact that many of the factors associated with creep in metals, such as the development of plastic strain, and the formation of cavities and their eventual coalescence to generate demonstrable crack-like defects, affect the EPD signals in much the same way that a developing crack would. However EPD usually comes into its own when most of the creep lifetime has been expended, and can show poor sensitivity to the earlier stages of creep damage development. Additionally, the transfer of laboratory based (i.e. well controlled) EPD set-ups to the more practical environments inherent in on-line plant life monitoring is fraught with difficulties, not least of which is interpreting the signals. Changes in the EPD can be generated by factors in the test environment that have little do with actual creep damage, such as those due to variations in test conditions (typically temperature and/or applied load). These changes can be orders of magnitude greater than those that are expected via creep damage alone.

This paper details some of the EPD work carried out as part of a series of long term creep tests performed on P91 and P92 pressure vessels. This is turn was part of a wider study aimed at better lifetime prediction for components manufactured from these materials and utilized in power generation applications. 
This paper reports on many of the practical issues and the lessons learned in undertaking this work, and in particular discusses the feasibility of applying the EPD technique into a real-life industrial on-line monitoring context.

\section{Background}

In its most basic form, EPD relies upon a measurement of a specimen's electrical impedance. In the case of DCPD, this is specifically the electrical resistance, whereas in ACPD, capacitive and inductive components complement the electrical resistance to generate a more complex parameter [3].

Conventionally, impedance is measured using a four point arrangement of inline electrical contacts, with the outer two connections delivering the excitation current, and the inner two allowing measurement of the potential drop required to drive the excitation current through the specimen (this being a function of the impedance). The method relies upon the influence of a defect on the specimen's impedance. Normally crack-like defects act to raise the local impedance, and therefore can be detected by a rise in the local EPD. Measurements are normally simplified by ensuring that the excitation current is known and remains constant at least throughout the duration of the measurement.

A further subtlety of ACPD over DCPD is the existence of the so-called skin effect, where the excitation current is found to travel close to the surface of the specimen, rather than uniformly throughout its cross-section (this being largely the case for DCPD). A practical consequence of this phenomenon is that the calibration 
methodology (EPD vs crack depth) is different for ACPD compared to DCPD.

Additionally the skin effect (specifically the depth that most of the current penetrates to - the skin depth) is a function of the frequency of the AC excitation, and this provides ACPD with an extra degree of freedom which can both add information or complicate interpretation (depending on one's viewpoint). The higher the frequency, the smaller the skin depth, and the more sensitive the technique is to surface breaking defects.

Many of the practical challenges associated with EPD relate to the engineering difficulties in maintaining a constant current, and also the way in which electrical connections are made to specimens [3]. However, EPD excels at providing a continuous electrical response proportional to crack dimensions and as such it is often the only crack monitoring technique that can be used in extreme testing contexts such as at high temperatures (e.g. thermomechanical testing of superalloys) [4], under corrosive atmospheres (e.g. $\mathrm{H}_{2} \mathrm{~S}$ induced cracking in the oil and gas industry, or stress corrosion cracking of stainless steels under high pressure high temperature aqueous conditions) [5], or even in high radiation environments (such as in-pile testing of materials in the nuclear industry). In such contexts EPD is normally used in a continuous (on-line) sense to monitor for crack initiation and crack growth.

For NDE applications, EPD equipment can be battery powered and then used for spot-checking of cracks in structures (particularly ACPD, given it's better portability), with the 4 point connections being made by some kind of position-able or hand-held "probe" head which usually houses sprung loaded pins able to penetrate surface oxides or contamination. However, resolutions reached (in terms of crack depth) are nothing like that achievable in an on-line context. This is as a result of several reasons. Very 
few commercial hand-held crack depth "meters" have ever employed DCPD as the measurement method, simply because most metallic specimens are very effective short circuits, so the excitation currents have to be in the 10's or even 100's of amps before a decent measureable DCPD can be obtained - and passing such high currents through sprung pins is not a very long term proposition. Therefore any DC based system is likely to be of poor sensitivity as a consequence of the low maximum current capability.

Conversely, handheld ACPD probes are very susceptible to registering changes in signal magnitude due to differences in approach angle and contact pressure. These effects are linked to the existence of an error signal, that superimposes upon the specimen-derived signal, and is generated as a consequence of induction phenomenon (much like lift-off in Eddy current based NDE). The resultant variability inevitably affects resolution.

Such effects generally limit handheld EPD to a resolution of no better than $0.5 \mathrm{~mm}$ in crack depth measurements - a far cry from the 10 micron resolution that is normally achievable for typical cracks in a continuously monitored situation [4]. Not only does this limit the utility of EPD based NDE of cracks, it does preclude probe based measurement of a range of other effects that might be of interest (and which have been shown to influence EPD readings) including microstructural differences (such as weld HAZs, or case hardening), internal defects (e.g. porosity in castings), residual stress measurements (ACPD is sensitive to the level of elastic and plastic strain in ferrous materials), and of course creep damage, such as the development of cavitation. 
EPD seems to have been rarely used for on-line use, and this may have something to do with the practicalities of making robust connections in the field as well as interpreting the data generated. In this study, many of the practical issues associated with specimen connection and apparatus deployment have been overcome - certainly to the extent which no longer requires a laboratory based or academic approach to be undertaken. Data interpretation remains a challenge, although as will be described here, initial results of the work on this look promising. Part of the advance has been to combine $\mathrm{AC}$ and $\mathrm{DC}$ variants of the EPD technique into one monitoring system to effectively create a "ACDC"-EPD set-up. Not only has this enabled the benefits and strengths of both variants to be captured in one on-line test, it has also revealed a synergistic effect which appears to greatly enhance an operator's ability to determine (in this case) how close to end-of-life a monitored component is.

\section{Practical details}

The ACDC set-up employed in this study was created by interfacing two commercial EPD instruments together, one providing ACPD capability and the other, DCPD, (Matelect Ltd, London). This was achieved by using a series of signal and current multiplexing (switching) units which also facilitated connection to multiple points on the test vessel. Several cylindrical pressure vessels were monitored, in a three year study. Figure 1 shows one of these - manufactured from P91 steel and containing two circumferential welds. The study aimed to investigate creep damage development, and to characterize this using a variety of non-destructive methods. The vessel was mounted vertically (Figure 1) and subjected to both elevated temperature (ca 700 DegC), internal pressure, and external axial load. The vessel was loaded to levels which, at the test temperature, were deemed to result in failure via creep in around 10k 
hours. Failure was expected to initiate in the HAZ of the welds by Type IV cracking and it was these zones that were monitored by the EPD system. Scheduled outages were also planned (as part of the wider study) so that an array off-line NDE characterisation methods could be employed. This included ACPD (in a hand-held mode), but this paper only discusses on-line results, as this methodology will ultimately prove more useful to an industrial user, than an outage based assessment of lifetime.

A significant feature of the ACDC system was the ability to employ only one set of electrical connections for both EPD variants. This both simplified connections and reduced the number of wires and connection points to the test vessels, the only disadvantage being that the ACPD current carrying wires were, in effect, much thicker than they would normally be (given they also had to be capable of carrying the higher direct currents for DCPD).

After a considerable period of trial and error, a connection methodology which involved the use of stainless steel studs (ca 20mm long x $2 \mathrm{~mm}$ diameter) silver-soldered to pure silver wire, the whole being sheathed in silica braiding, was eventually employed. The studs were welded to the vessels using a conventional spot welder, operated as a stud welder.

Studs were pre-tinned to assist subsequent soldering, and in some cases, they were also pre-soldered to short $(100 \mathrm{~mm})$ lengths of silver wire, which proved easier to join in the field to the main silver leads, especially where access was limited. The silica braiding was applied to pre-measured lengths of silver wire before soldering. Wire of $1.5 \mathrm{~mm}$ diameter was used for the current supply lines, and 0.5 for the signal lines. 
Typical lead lengths were 3 metres from connection point on the test vessel to a nearby junction block (external to the furnace), whereupon connection to the multiplexers (and thence to the AC and DC instruments) was via shielded twisted pair copper cable. Wherever possible (and to help eliminate interference or "pick-up" in ACPD situations) all wire pairs (signal as well as current) were twisted together, although this was minimised to avoid damaging the silica braid. A weld contains two adjacent HAZ locations, and therefore each HAZ was monitored separately. A total of 6 studs were positioned in-line across a weld therefore, with the outer two delivering the requisite EPD excitation current, with the two inner pairs, straddling each HAZ, acting as the EPD measurement points. Several "zones" along any one weld were covered, and the complete ACDC system contained 18 separate EPD measurement points per vessel.

Part of this set-up and the P91 test vessel can be seen in Figure 1.

Silver proved an inspired choice, being extremely easy to handle in the field, thread through the braid, and solder in place. It resisted oxidation admirably, and even after 10,000 hours of exposure, was always bright and free from oxidation, unlike the stainless studs which became heavily oxidized. Connection failures were common at the start of the work, but once the pre-tinning was introduced, and the stud welding parameters optimized, these disappeared. Use of standard fluoride based fluxes for insitu soldering did give rise to degradation of the silica braid if residues were not scrupulously washed off, but with care, and the use of pre-tinned studs (which largely precluded in-situ fluxing), this problem was eliminated. Results using nickel wire were nowhere near as reliable and the higher electrical resistance of nickel required thicker wires to be employed (for the current supply leads) which hampered installation. 
The ACDC instrumentation was placed external to the furnace and blast zone, and the whole EPD system was placed under the control of bespoke software which could be accessed across the Net, and permitted the easy transfer of data for regular interpretation.

For the ACPD aspect, skin depth calculations for P91 steel at the frequencies available suggested that even at the lowest operating frequency of $300 \mathrm{~Hz}$, the skin was substantially thinner than the specimen wall thickness (ca $5 \mathrm{~mm}$ compared to $25 \mathrm{~mm}$ ). This meant that the ACPD readings were only expected to reveal defects and/or microstructural variations that were close to the outer surface of the pipe specimen. Creep cavitation could extend to the surface regions but past experience suggested that crack development would initially be internal before travelling to either the outside surface or the inside (back-face). The lowest excitation frequency $(300 \mathrm{~Hz})$ was therefore expected to give the best chance of showing any internal or back-face defect.

The ACPD system utilized was capable of providing both real and imaginary components of the ACPD reading. AC signals contain both phase and amplitude information, hence can be resolved into vectors representing the real (i.e. resistive) and the imaginary (capacitive and inductive) components of the signal. The resistive component is normally related to crack depth through a linear or near-linear relationship, whereas the imaginary component tends to be ignored in conventional ACPD. The imaginary component can however be used to help optimise the measurement set-up. If the values of the imaginary components are large, then this 
casts doubt upon the validity of the resistive components as saturation of the amplifiers in the ACPD system may well be occurring.

The AC excitation current was set to 2 amps for all measurements. In contrast, direct currents of ca 50A are sometimes required for comparable signal magnitudes, but this is often not possible using commercial equipment if the lead lengths are long (and hence the overall resistance of the current path). High currents can also cause specimen heating and this can (and did) lead to drift in DC signals if currents higher than 15A were employed. A similar set-up was employed for the P92 vessel that was an additional part of the study, the only differences being that this vessel was mounted horizontally, and no external axial load applied. Also, temporally, the P92 tests came after the P91 tests.

\section{Results and implications}

Testing of this kind creates vast amounts of data, and detailed analysis is not attempted here, however broad lessons for creep crack development and subsequent monitoring can be drawn, and will prove valuable to future studies. Figure 2 shows a plot of data extracted from a representative data file and illustrates a typical DCPD response over a total period of just over 2.5 months from an adjacent pair of HAZ locations (designated " $\mathrm{x}$ " and " $\mathrm{y}$ ") on the P92 vessel.

It can be readily seen that there is much variation in signal magnitudes over time. Note that the complimentary ACPD response would be equally as "busy". 
The fluctuations seen amount to several 10's of \% of full scale, and this is far in excess of any fluctuation expected due to crack initiation. Once a crack had initiated and grown to be a substantial fraction of the specimen wall thickness, such a change in signal level might be expected, and indeed exceeded, but clearly the observed fluctuations in Figure 2 recover in magnitude and level, and are definitely, therefore, not due to specimen cracking. Furthermore such changes were often observed early on in the projected lifetime of the vessel, so could not be reasonably ascribed to cracking.

Most of these fluctuations were traced to changes in specimen temperature. The two large dips (note dips, not rises as would be expected if a crack had developed) were actually associated with a total failure of the furnace temperature control and subsequent shutdown of heating.

In laboratory based EPD, during elevated temperature testing, it is normal to employ a reference channel to normalise for such temperature fluctuations. A ratio of active/reference EPD is then recorded and this should be immune from changes in temperature. Normalisation does indeed generate a far quieter response, however as Figure 3 shows, a simple arithmetic normalisation (in this case the signal from one HAZ (x) divided by the signal from the adjacent HAZ (y)) can still be fooled.

The presence of the transient in the normalised signal can only be explained if the effect of temperature on the signals is non-linear and/or the shutdown affected the signals via other, additional, (and non-linear) factors, such as a change in stress. 
The above issue highlights the difficulty and challenges that technicians will face when using EPD for on-line monitoring. Other signals (such as temperature) clearly need to also be monitored and interpreted alongside the EPD data, if any certainty is to arise in practice.

Irrespective of the above, it will still be necessary to "extract" a signal from such a response that in some way represents the information on creep behaviour. Once a transient can be explained in terms of an external factor, it is not unreasonable to simply delete the transient data, and re-join the ends of the cleaned response together. Other filtering methods can be employed to remove solitary transients which are clearly "rogue" points and act to mask the longer term trends. Figure 4 illustrates what can be done using this approach, to the data from the P92 vessel.

The data is now far noisier than before, as autoscaling has been employed to essentially raise signal gain (possible now that the transients have been removed). The scale now reveals that noise in the EPD is at a level of 10's of nanovolts - and hence is more likely to be a reflection of overall instrumental noise. Emerging from this noise can be seen a clear trend however - a gentle but steady rise in the EPD which is much more likely to be as a result of the development of creep damage than to "external" influences. Given that this data set is obtained from a zone on the P92 vessel directly over where final rupture occurred, more will be said about this rise later.

In the present study, once such data processing was performed on the $\mathrm{AC}$ and DC signals, further trends were identified, which helped to indicate that creep damage had reached the late stages in a specimen's lifetime, where cracking was expected. 
Figure 5 reveals what was observed to happen to the EPD once a defect had initiated in one of the circumferential welds, and was actively growing, in the P91 vessel. The presence of the crack was confirmed after catastrophic failure of the vessel had occurred. In this case, a rise in EPD was not being employed as an early predictor of impending failure, but an examination of the trends seen revealed a pattern of responses that was not entirely as expected.

It should be noted that, as before, two signals were being monitored at this zone, namely one from either side of the weld, so that both HAZ were covered (also AC and DC). The DC trends appear to work in opposition to each other with the one HAZ zone showing a clear exponential rise associated with a rapidly propagating defect, but the complimentary HAZ trace shows a gentle decline. The explanation for this is easily understood if it is noted that both monitored zones are being fed the same excitation current and lie in line with each other (in terms of current flow) hence the growing defect will a) raise the DCPD (in the expected sense) and b) divert the current flow away from the adjacent zone such that it appears to show a reduction in measured DCPD.

It was clear that the change in the DCPD was very dramatic and highly definitive, when in the latter stages of failure (the last two days), but that nevertheless, a far subtler rise was seen at least two weeks ahead of failure. Given the comments made earlier, such a rise would have been insufficient to provide an operator with any great certainty of impending failure - at least until a few days before it occurred. 
However, when considered together with the drop seen in the adjacent HAZ's response, a greater degree of certainty could be assumed whenever a similar "pattern" is observed.

Further to this, Figure 6 illustrates the corresponding ACPD traces, for the same locations on the P91 vessel, and here it can be seen that as the DC traces rise, the AC responses drop. Unlike for DCPD, this occurs on both HAZ positions and the drop continues until such time as the DC trace has begun its exponential rise, at which point the AC response also turns and appears to follow suit by rising almost vertically (signifying rapid crack propagation).

The explanation for the $\mathrm{AC}$ behaviour is not obvious and relies upon the knowledge that in ferritic materials, ACPD signals are sensitive to stress (strain in reality) [1]. This is a result of the change in magnetic permeability that occurs when the grains containing the magnetic domains are strained. As a result, in a ferritic material under a uniaxial stress, the ACPD measured axially has been observed to drop as the stress rises.

In the monitored pressure vessel, the developing defect will be expected to raise the local stress to a point where the ACPD may indeed be affected by the stress concentration.

Given that the "true" ACPD is normally only sensitive to surface breaking defects (as it relies upon the skin effect to generate a rise in path length as a defect 
grows) it is likely that the $\mathrm{AC}$ response will first drop before finally rising (presumably once the defect has become surface breaking).

When taken together, the drop in $\mathrm{AC}$ with the rise in $\mathrm{DC}$, and the drop in $\mathrm{DC}$ on one HAZ, with a rise in the other, constitute a characteristic "signature" which could greatly lengthen the warning period ahead of an impending failure.

After this initial success, the failed weld in the P91 vessel was removed and the shortened vessel was placed back under test, with the aim of enabling the second weld to fail. The ACDC EPD monitoring system was re-instigated with fresh connections around this remaining weld. Similar trends were noted in some of the monitored locations in this second test and, as these trends were developing in time, a decision was taken to eventually stop the P91 creep test ahead of a scheduled outage. No surface breaking defect was observed (and none was found subsequently in the internal surfaces of the vessel), but ultrasound inspection (UT) was implemented and confirmed the existence of sub-surface cracking. Subsequent sectioning and metallographic examination has supported the UT and EPD results, and has revealed that as well as an internal crack, substantial cavitation damage had occurred in the vicinity of the developing defect. However the possibility remains that the cavitation is an effect of, rather than the cause of, cracking.

Conservative estimates of the length of warning that the EPD gave the test operators of a leakage in the P91 vessel, via a surface breaking defect, are in the region of two to three weeks. This may well be enough for many plant operators. 
Unfortunately, the difficulty with definitively ascribing changes in the observed EPD to creep damage per se, (i.e. other than to cracking, as initiated, through creep), is that none of the tests conducted managed to catch a test vessel before evidence of cracking had developed (albeit internal cracking). Thus, maintaining that EPD can detect creep damage (as distinct from cracking), such as a rise in local strain and/or the development of cavitation, is easy to challenge. In truth, whilst the ability to see such damage is clearly of value to metallurgists and researchers alike, its value to plant operators is probably less than a reliable warning of impending vessel failure. Nevertheless, in defence of the belief that EPD can indeed be sensitive to pre-crack damage, Figure 4 should be recalled.

The subtle (relatively), but steady, rise in DCPD in Figure 4 was actually present for some 2.5 months (ca. 1600 hours) before the test was terminated and the P92 test vessel examined using UT. No crack-like defects were detected, the EPD connections remade, and the vessel was put back under test. The gentle rise in DCPD was observed to continue (at a similar, almost linear, gradient) until final cracking and failure occurred some 2 months later, whereupon a rapid rise in DCPD was observed (similar to that seen in the earlier P91 tests). The percentage change in DCPD amounted to less than a $1.5 \%$ rise over the initial 4.5 months, whereas the change in the last few hours of life amounted to over $100 \%$. It is highly unlikely that a crack-like defect existed 4.5 months prior to failure, so the subtle change in DCPD may well have been due to "other" forms of creep induced damage, for example cavitation.

The longevity, expense, and complexity of the vessels tests limited the ability to reproduce these observations, but of the three tests conducted to failure (2x P91 and 1x 
P92), all three have shown modest initial rises in DCPD readings close to the ultimate failure location. Obviously, well defined and constructed laboratory tests would provide a better understanding of the influence of creep on the EPD response in P91/P92, in contrast to the real-word tests conducted here, but this should not detract from the positive nature of the results.

\section{Conclusion}

We present here encouraging results for a modern manifestation of a traditional technique. The EPD work carried out as described has led to a substantial improvement in the practical knowledge of how an EPD based monitoring system should be implemented on an "industrial" creep test specimen, in particular, for helping to detect the later stages of life and the onset of final fracture and cracking. It is envisaged that transferring this experience and methodology to in-situ testing of power station components in service would not pose too great a challenge.

What has always been presented as a challenge has been the interpretation of the results, however. This has traditionally been the domain of the scientific researcher rather than staff working in an engineering and maintenance context. This is due to the complexity of the signal responses and the likelihood that changes in signal can be ascribed to a range of phenomena, only some of which have anything to do with creep damage.

This situation has not been totally eliminated by the current work, but the emergence of the notion that a combination of ACPD and DCPD can help in interpreting creep behaviour, is a definite step forward. This notion was based on the 
idea that a developing defect, such as a crack, will increase specimen resistance (hence raising the $\mathrm{PD}$ ) in the case of $\mathrm{DCPD}$, but could also raise section stresses (due to the development of stress concentrations) hence lowering ACPD (up to a point, and then the PD would rise, as the contribution from the defect began to dominate).

Of note in the case of DCPD, was a further synergistic effect gained by the use of pairs of signal pick up points (one for each HAZ of a monitored weld), which revealed that as the one straddling the developing defect increased, the one on the complimentary HAZ reduced. This can be explained by the developing defect's additional ability to redirect the flow of excitation current away from the second HAZ measurement point. The consequence of a lower current density was a lower signal magnitude - so as one signal rose, the other fell.

In addition to helping interpret the on-line results, the idea of a "signature" for defect detection, if correct, could allow earlier detection - much earlier that would be the case if a single EPD response was being monitored.

It should be noted that the signature described here is particularly confined to a double HAZ situation in a weld in a ferritic material, however it is feasible to suggest that other signatures can be identified and checked against, in other testing contexts.

Of further note were EPD observations that were made well before ultimate failure of the test vessels. These showed that once larger transients were stripped out, locations where failure subsequently developed did show subtle, but definite, changes in EPD months prior to rupture. In particular, DCPD responses were seen to rise in a linear 
fashion for up to 4.5 months before final fracture. The challenge now is to catch another vessel "in the act" well before any demonstrable cracking has developed.

\section{Acknowledgements.}

The authors would like to thank Dr A Shibli and Dr D Robertson of ETD Consulting of Leatherhead UK for their help and support in undertaking the work described here and MPA Stuttgart for access to their facilities and universally helpful staff (especially Dr A Klenk and Dr A Hobt).

\section{References}

1. Venkatsubramanian, T. V., Unvala, B. A, 1985, An AC potential drop system for monitoring crack length, Journal of Physics E: Scientific Instruments, 17(9) pp765-771

2. Gibson, G. P., 1987, The use of alternating current potential drop for measuring Jcrack resistance curves, Engineering Fracture Mechanics 26(2) pp213-222

3. Annex A6, 2015, Guidelines for electrical Potential difference determination of crack size, ASTM E647-15e1 Standard Test Method for Measurement of Fatigue Crack Growth Rates, ASTM International

4. Marchand, N. J., Dorner, W., \& Ilschner, B., 1990, A Novel Procedure to Study Crack Initiation and Growth in Thermal Fatigue Testing, ASTM STP 1060 pp237-259. 
5. Fanjiang, M., Lu, Z., Shoji, T., Wang, J., Han, E., Ke, W., 2011, Stress corrosion cracking of uni-directionally cold worked $316 \mathrm{NG}$ stainless steel in simulated PWR primary water with various dissolved hydrogen concentrations, Corrosion Science 53 pp2558-2565 


\section{Figures}

Figure 1. In-situ set-up on the P91 test vessel (EPD leads are visible as white wires exiting to lower RHS).

Figure 2. Typical DCPD plot for period of approximately 3 months showing massive fluctuations associated with changes in thermal conditions. Data is for two adjacent but in-line HAZ monitoring zones, designated " $\mathrm{x}$ " and " $y$ " on the P92 vessel.

Figure 3. Normalised DCPD plot for data presented in Figure 2 (thus ratio HAZx/HAZy) which should have eliminated any changes due to temperature fluctuations, but clearly has not.

Figure 4. Processed DCPD plot for early stage data from the P92 vessel (HAZy only) at the location where final failure occurred. Transients have been removed and the vertical scale expanded. Long term trends can now be seen at or close to the intrinsic noise level of the technique, and are several orders of magnitude smaller than transients seen previously.

Figure 5. DCPD plot for zone containing a developing creep crack on the P91 vessel of Figure 1. Rising curve was for crack site HAZ, whereas falling curve for adjacent noncracked HAZ. (x-axis represents 1 month approx.).

Figure 6. Corresponding ACPD plots for zone in Figure. 5 containing a creep crack. Both traces steadily drop (ignoring transients) until crack rapidly propagates to final rupture. 
Fig1

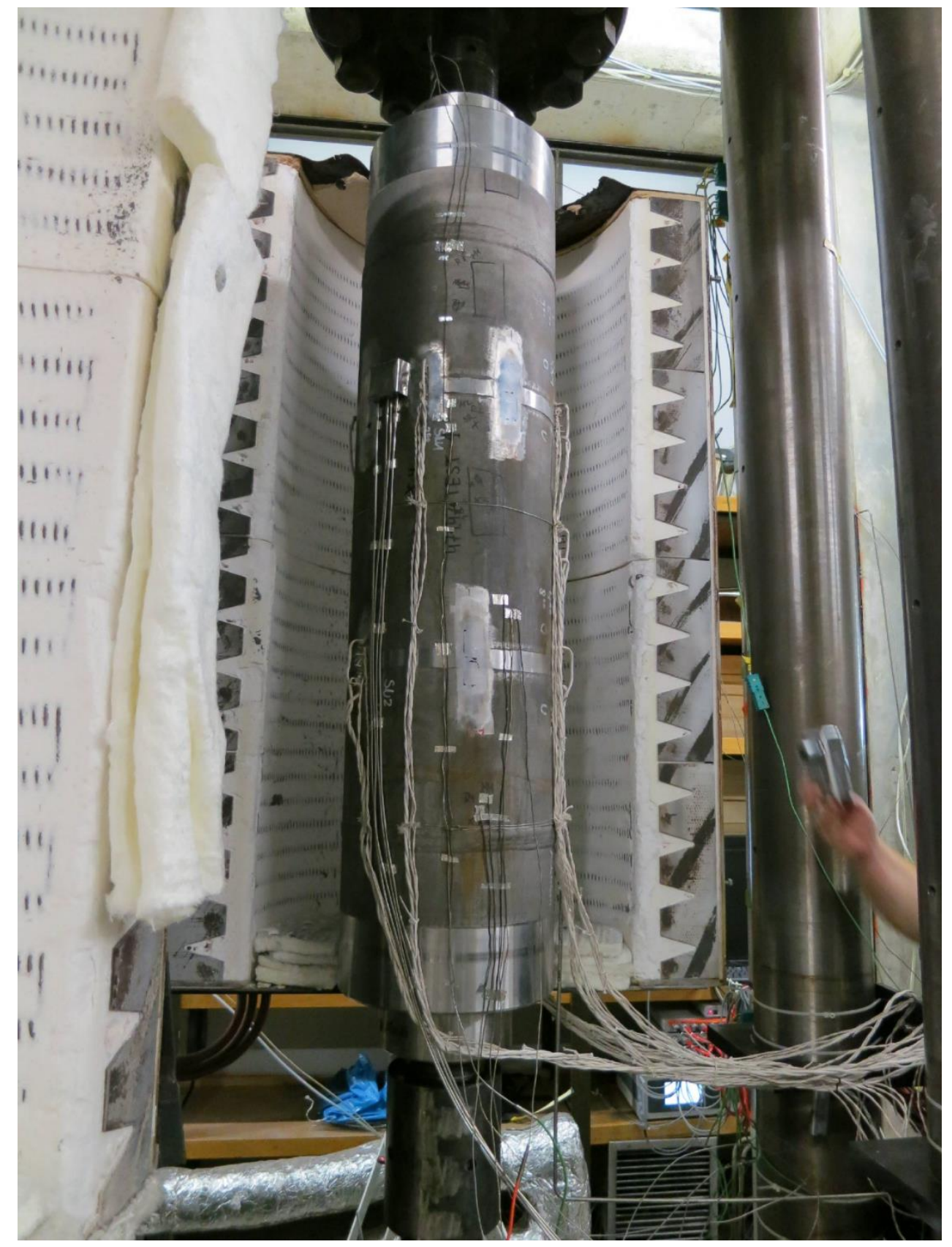


Fig 2

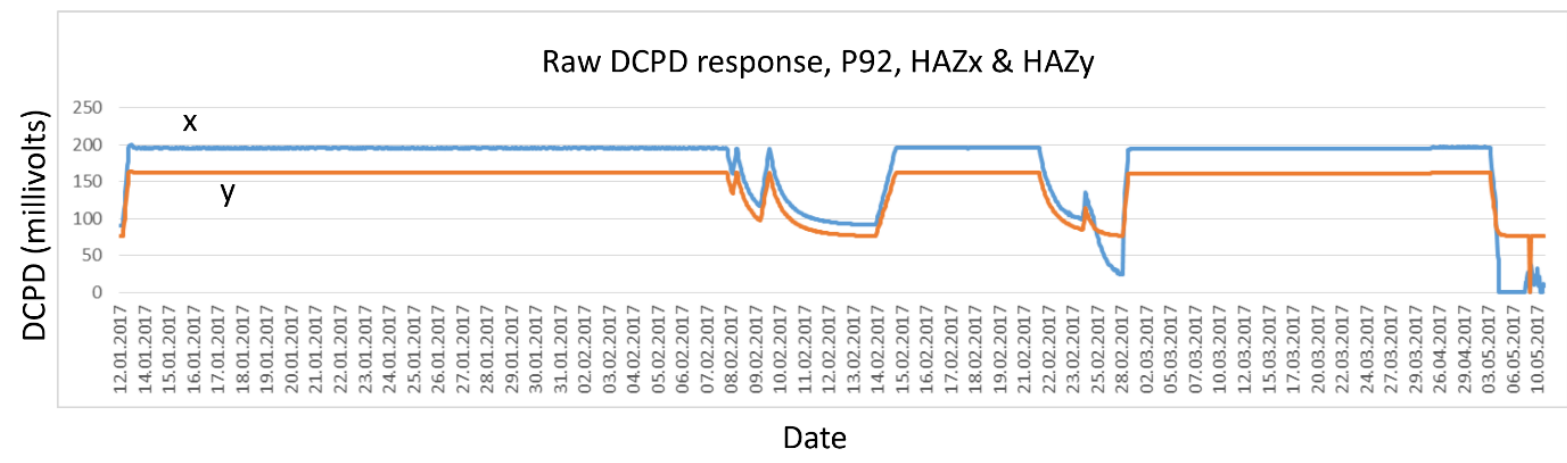

Fig 3.

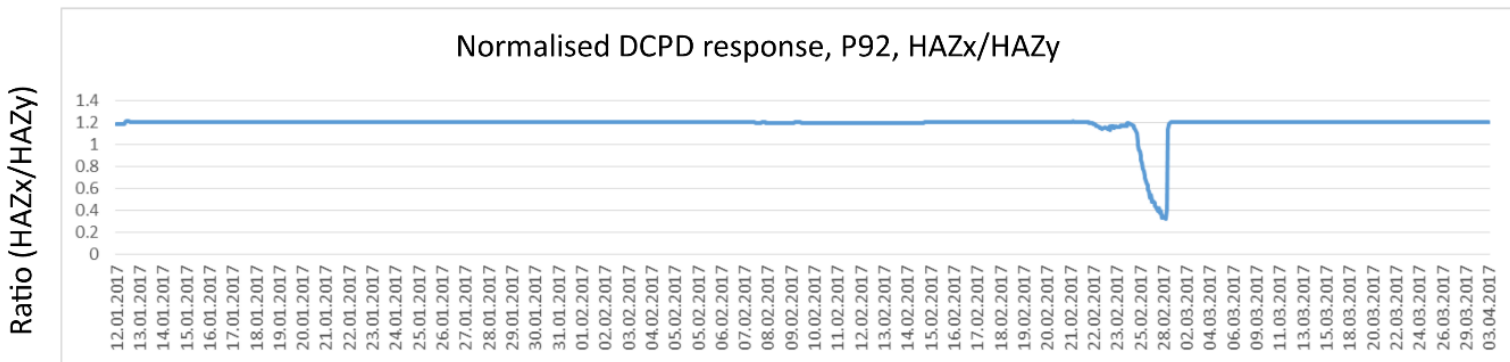

Date

Fig 4.

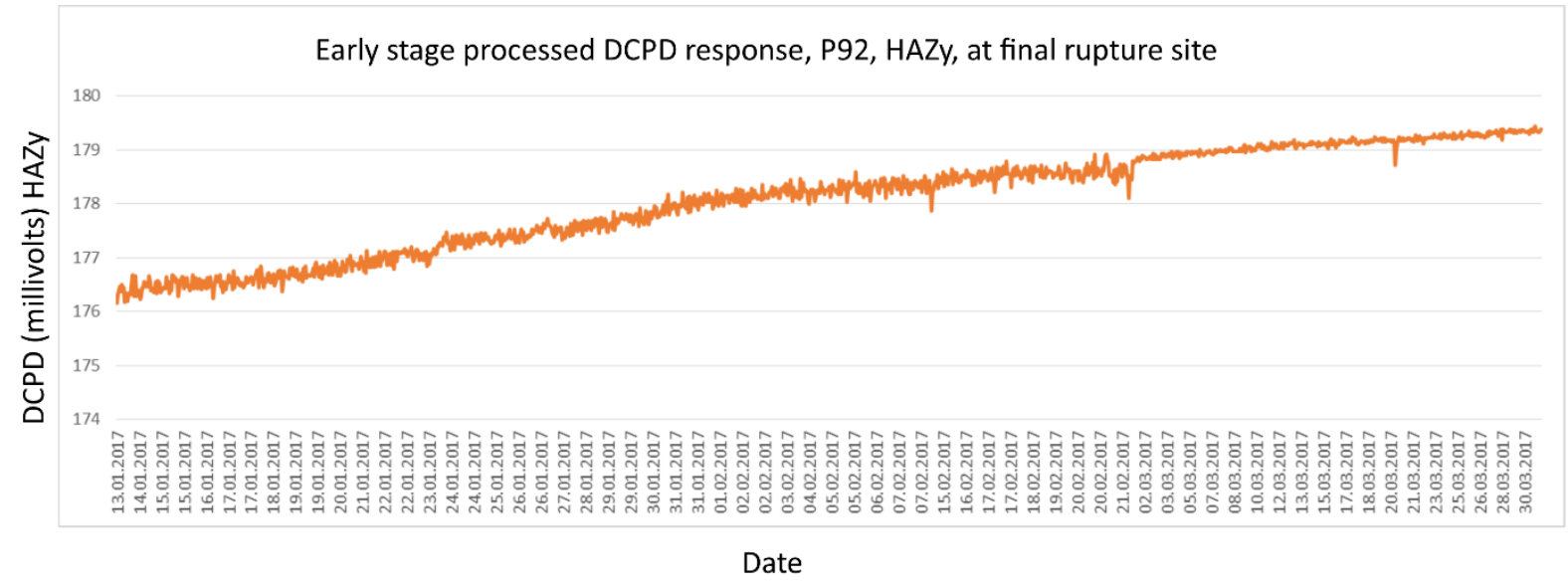


Fig 5.

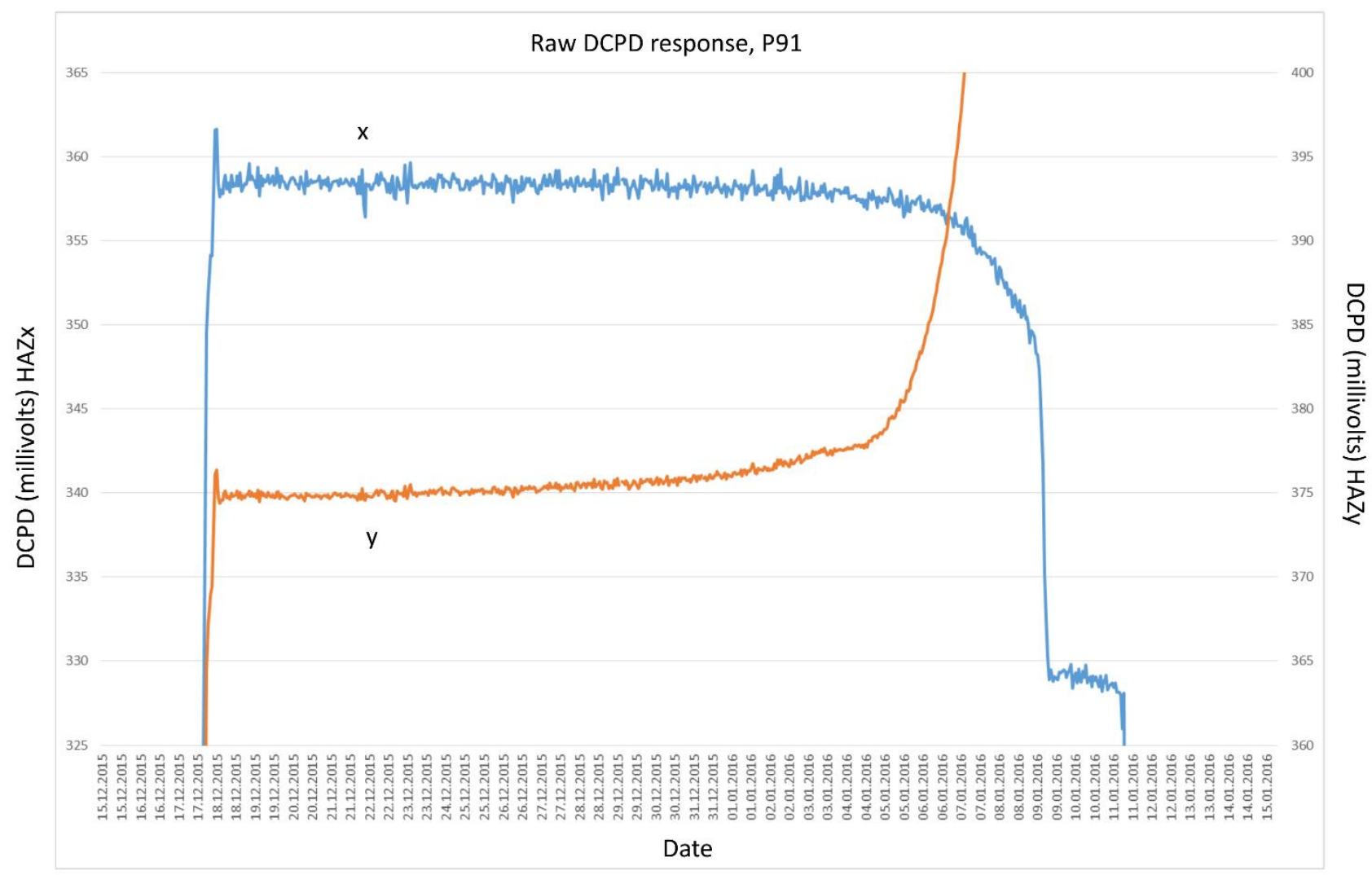


Fig 6.

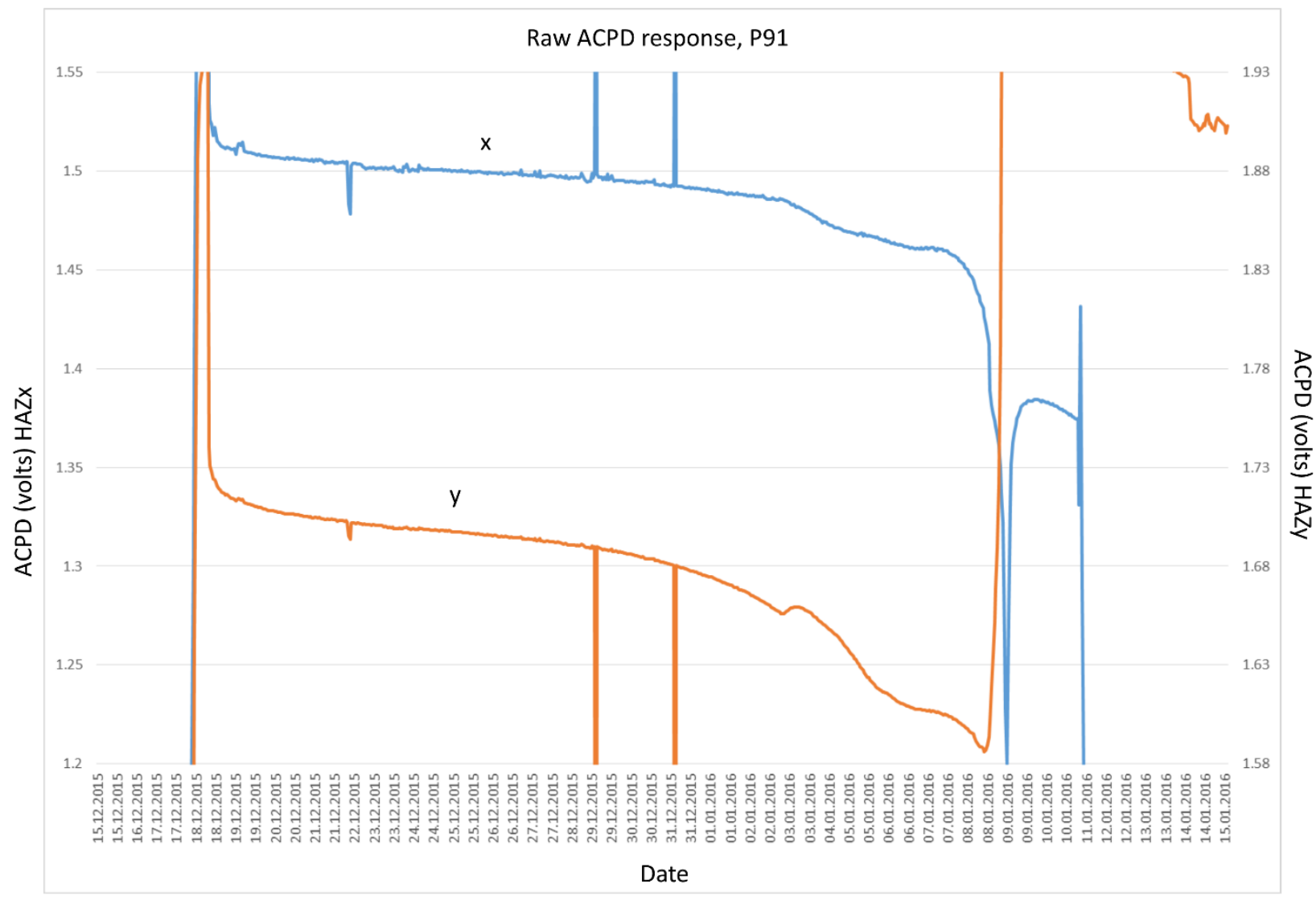




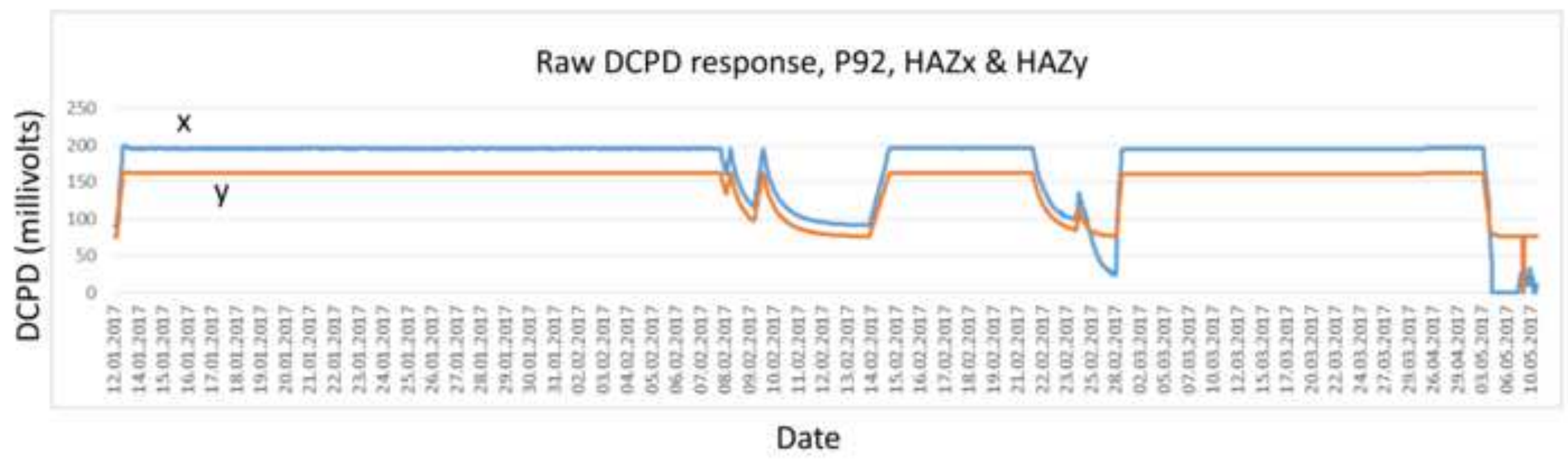




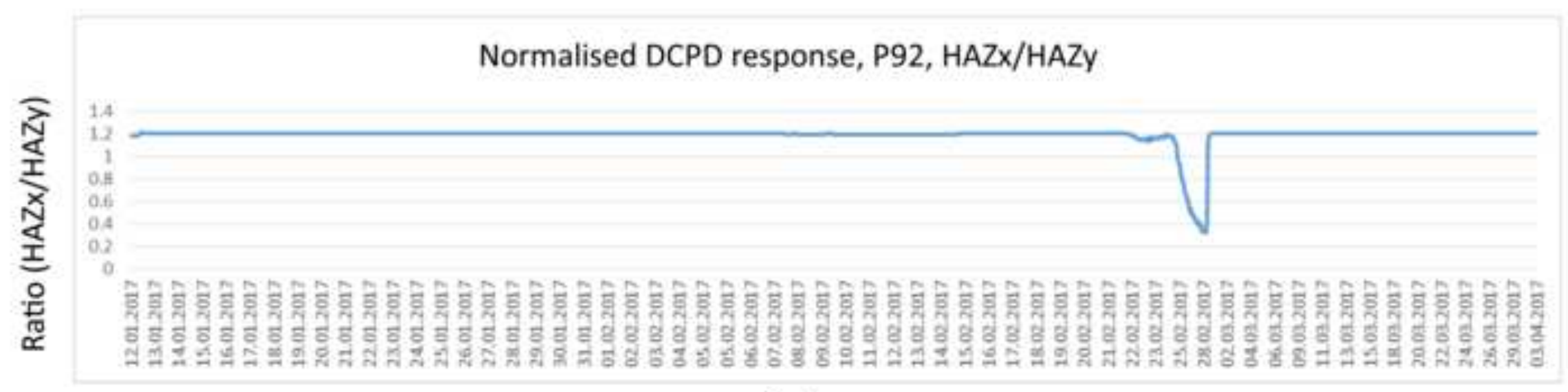

Date 


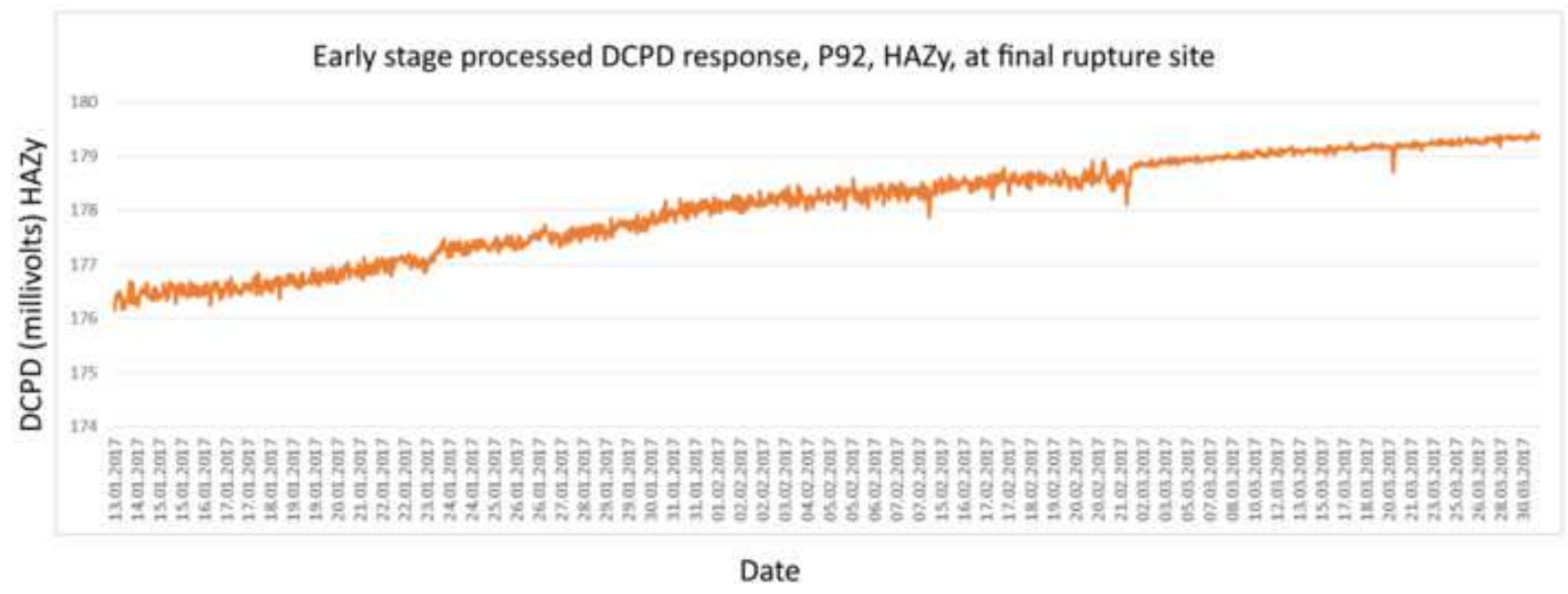




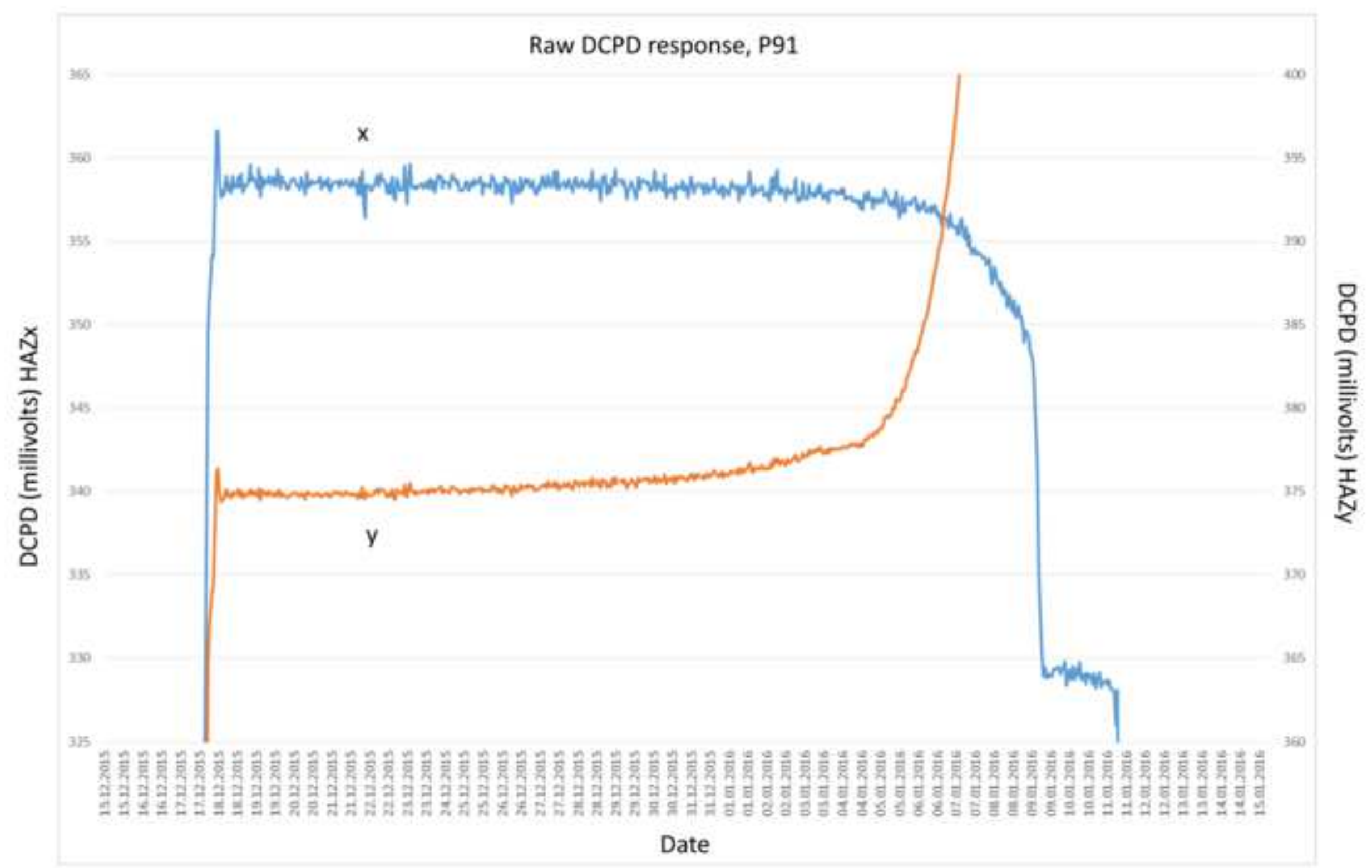




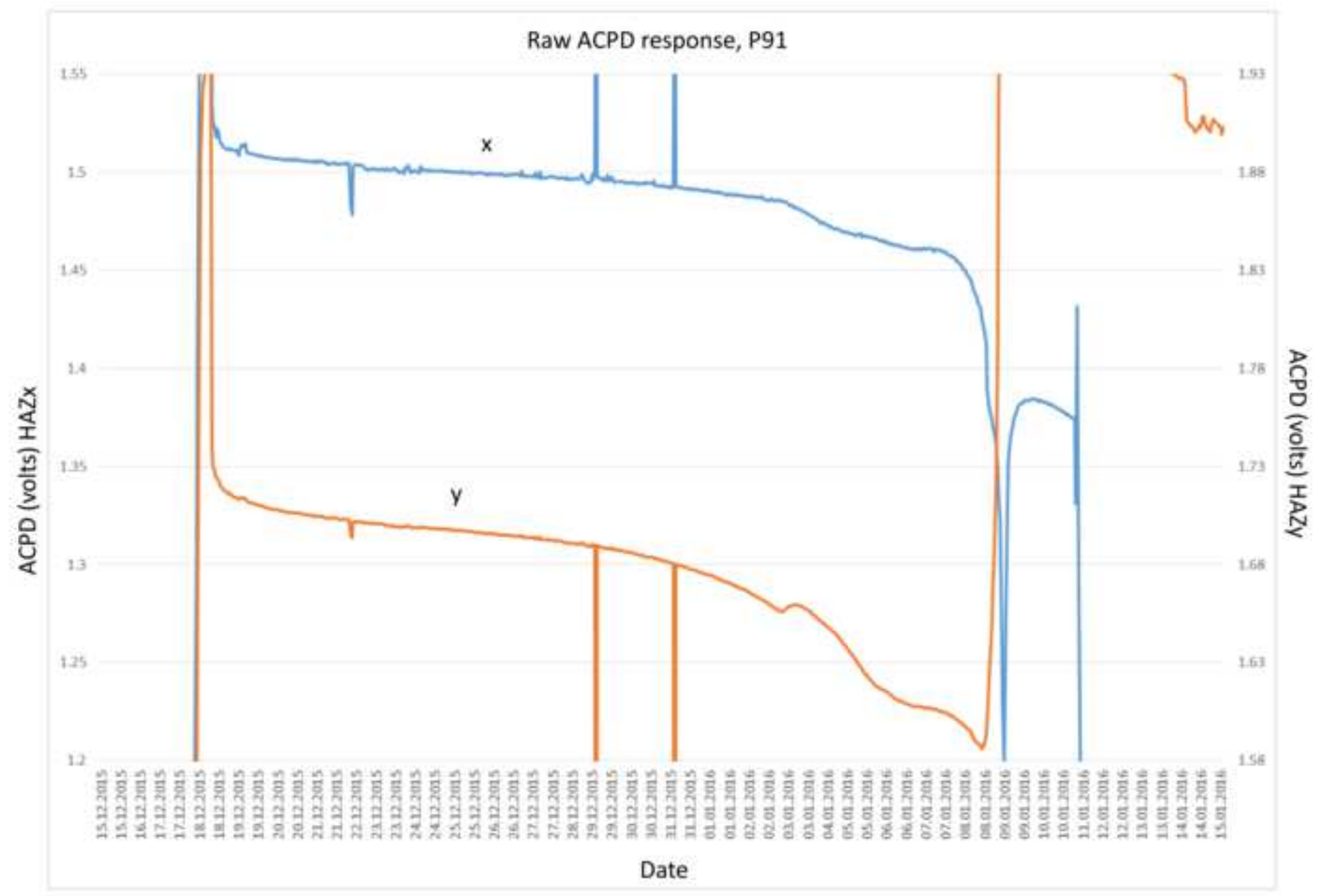




\title{
Responses to reviewers comments: Manuscript MHT536 September 2017, Adam Wojcik
}

\author{
Reviewer 1 \\ This is a very interesting paper, and worthy of publication, but should address the following points: \\ - There is an assumption that the reader is familiar with the Matelect DCPD unit (e.g. Fig. 2). The \\ authors assume too much of the reader. For example, please clarify what is meant by ' $x$ ' and ' $y$ ' \\ - No physical evidence is given to substantiate and validate the interpretation of the Matelect output. \\ In view of the title of the paper, this is a significant omission and should be rectified, for example with \\ sample photomicrographs.
}

See also below for $2^{\text {nd }}$ Reviewer:

The revised manuscript addresses the confusion associated with the $X$ and $Y$ nomenclature. This is a justified comment and the terms have either been fully explained or eliminated where they might cause confusion. Similarly in the captions to the illustrations and on the illustrations themselves.

See also below for the $2^{\text {nd }}$ Reviewer:

In terms of justification for the interpretation of the EPD data, again the reviewer's (both) comments are accepted. The tests carried out, however, were on real-life rather than laboratory specimens, so the opportunity to carry out such additional testing was limited. Clearly cracking was deemed to have occurred (as stated), given the vessels eventually failed. There would not be much academic utility in showing a vessel that has clearly ruptured. Where sample micrographs should ideally have been included is in order to assist with the evidencing of pre-crack damage - of the type associated with the earlier stages of creep based failure - such as cavitation. Independent measurements of surface and near surface cavitation were performed by a partner organization (in-situ measurements, so quite limiting), and this did show some evidence of cavitation well before final rupture, so the authors are confident that the EPD can respond to such changes. In reality, however, the vessel tests were not ended well before rupture, and it would have been much better had they been done so, so that the very subtle changes in EPD seen after the results were post-processed, could be better ascribed to a pre-cracking phenomenon. In defence of the assertion that EPD can see creep damage, as opposed to cracking (which is beyond doubt) is the fact that very subtle rises in EPD (particularly DCPD) were observed for a number of locations (all of which turned out to be the final rupture sites - or very close to), well before any cracking could reasonably have been expected to occur (and indeed well before any suspicion of cracking or a positive UT examination). In some cases, such rises were seen many months away from final failure. These can only be ascribed to non-crack-like mechanisms. On-going work seeks to stop a vessel test at this stage, so that a more academic appraisal and study of damage mechanisms can be achieved. The authors have accordingly added a full explanation of this situation into the manuscript and hope this will satisfy the reviewers.

\section{Reviewer 2}

The Abstract implies that an EPD technique has been developed capable of detecting creep damage. The evidence given in the main text does not substantiate this. The words are misleading, and should be modified.

Ditto the response given above. Yes, a justified comment, but there are reasons for the apparent omission on our part. We did not intend to mislead. The nature of the test (given it went to completion) and the nature of the specimen meant that evidence of creep damage development other than via cracking would always emerge after final rupture - thus making any evidence of dubious value. 
For example, evidence of cavitation was seen in post failure specimens, but no one could say whether this was caused by the crack or caused the crack. Nevertheless the authors suggest that enough evidence of pre-crack EPD responses have been presented to justify not changing the title of the manuscript - which both covers both the pre- and post-crack stages of creep. In particular, plant operators have welcomed the ability of EPD to provide late information of developing defects even at the later stages of lifetime. It is acknowledged that from an academic viewpoint, however, earlier indications might also be valuable, and this deficiency will be addressed in future work.

Practical Details (page 6), line 37: The way in which reference is made to the two Matelect Unit identifications, and to Matelect borders on commercial promotion, but is probably acceptable. MHT editorial management should assess if there are implications relating to this sentence

We have simply reported the model number and the manufacturer of the mission-critical apparatus that we used for the study, as would be expected in most experimental sections of a journal paper. Nevertheless we accept the reviewer's concern and have accordingly edited the entry to just detail the manufacturer.

Results and Implications (page 10), first paras: While the text gives the impression that Figure 2 illustrates a typical DCPD response and a typical ACPD response (in the first and second paras of this section), the heading of Figure 2 implies that both records shown are DCPD at different locations. The legend is inadequate and unhelpful unless one is familiar with the Matalect DCPD equipment. The reference to ' $x$ ' and ' $y$ ' means nothing unless one is familiar with the way the logic adopted by the Matalect DCPD unit. This confusion should be rectified in the main text and/or the figure heading/legend. Most importantly, appropriately clear and understandable legends (and/or headings) should be adopted for Figure 2 and subsequent figures

Ditto the first reviewer. Thank you for this comment - we fully acknowledge the lack of clarity in how the labelling was presented and have addressed this aspect fully. There should be no evidence remaining of proprietary nomenclature, and the relevance of $X$ and $Y$ have been fully clarified in the manuscript in several locations.

Relating to the text in the middle of page 11, the temperature sensitivity provides a serious challenge for in-service measurements, because of inevitable thermal gradients and thermal transients (which do not exist to a significant extent in the experimental setup shown in Figure 1). This should be acknowledged in an addition to the text.

We absolutely agree here, however a full and comprehensive acknowledgement of this issue was already present in the manuscript. For example on page 12 beginning "The above issue highlights the difficulty...." It is difficult to see how much more definitive we can be, although we have nevertheless added to the manuscript to emphasize the challenges that face plant operators in predicting and/or detecting failure via on-line monitoring.

Page 12, line 19: replace 'weld' with 'welds'

Done, thank you. Date was also corrected elsewhere to data!

Page 15, line 2: The paper would have been much stronger and far more convincing if examples of the results of metallographic examination had been included. It is strongly recommended that at least one example is included in the paper if possible. After all, the title of the paper is "the use of potential drop technique for creep damage monitoring ..." In view of the lack of metallographic evidence and the statement in parentheses in line 2, it is recommended that the title is changed. It is currently misleading. While EPD has the potential for use as a technique for creep damage Monitoring, it is not demonstrated in the paper. The text should be modified to reflect this. 
Ditto the first Reviewer. Absolutely agree here, and hopefully the explanation given above, as well as in the revised manuscript will satisfy both reviewers. We would prefer to leave the title unchanged, as it does suggest the work is a) aimed at the industrial application, b) a technique for creep crack development (end of life) first, with creep damage second. We do however provide some encouraging evidence for the latter and have amended the manuscript to reflect this. We have added an acknowledgement that more work on this would be sensible.

There is no date to reference 1

Thank you - that has been corrected. 